\title{
AN ADAPTIVE EDGE ELEMENT METHOD WITH PERFECTLY MATCHED ABSORBING LAYERS FOR WAVE SCATTERING BY BIPERIODIC STRUCTURES
}

\author{
GANG BAO, PEIJUN LI, AND HAIJUN WU
}

\begin{abstract}
An edge element adaptive strategy with error control is developed for wave scattering by biperiodic structures. The unbounded computational domain is truncated to a bounded one by a perfectly matched layer (PML) technique. The PML parameters, such as the thickness of the layer and the medium properties, are determined through sharp a posteriori error estimates. Numerical experiments are presented to illustrate the competitive behavior of the proposed adaptive method.
\end{abstract}

\section{INTRODUCTION}

We consider the prediction of the scattered modes that arise when an electromagnetic wave is incident on some biperiodic structure. The electromagnetic fields in the whole space are governed by the following time harmonic (time dependence $\left.e^{-\mathbf{i} \omega t}\right)$ Maxwell's equations:

$$
\operatorname{curl} \mathbf{E}-\mathbf{i} \omega \mu \mathbf{H}=0, \quad \operatorname{curl} \mathbf{H}+\mathbf{i} \omega \varepsilon \mathbf{E}=0 .
$$

Here $\mathbf{E}$ and $\mathbf{H}$ are the electric field and the magnetic field, respectively. The physical structure is described by the dielectric permittivity $\varepsilon(x) \in\left(L^{\infty}\left(\mathbf{R}^{3}\right)\right)^{3 \times 3}$ and magnetic permeability $\mu(x) \in\left(L^{\infty}\left(\mathbf{R}^{3}\right)\right)^{3 \times 3}, x=\left(x_{1}, x_{2}, x_{3}\right)^{\mathrm{T}}$. The dielectric permittivity $\varepsilon(x)$ and the magnetic permeability $\mu(x)$ are assumed to be periodic in the $x_{1}$ and $x_{2}$ direction with periods $L_{1}$ and $L_{2}$, respectively:

$$
\begin{aligned}
& \varepsilon\left(x_{1}+n_{1} L_{1}, x_{2}+n_{2} L_{2}, x_{3}\right)=\varepsilon\left(x_{1}, x_{2}, x_{3}\right), \\
& \mu\left(x_{1}+n_{1} L_{1}, x_{2}+n_{2} L_{2}, x_{3}\right)=\mu\left(x_{1}, x_{2}, x_{3}\right),
\end{aligned}
$$

for all $x_{1}, x_{2}, x_{3} \in \mathbf{R}$, where $n_{1}, n_{2}$ are integers. Assume that $\operatorname{Im} \varepsilon(x)$ is a positive semi-definite matrix and that $\operatorname{Re} \varepsilon(x)$ and $\mu(x)$ are positive definite matrices. Assume also that the medium is homogeneous away from the region $\left\{\left(x_{1}, x_{2}, x_{3}\right)^{\mathrm{T}}\right.$ :

Received by the editor March 19, 2008 and, in revised form, January 1, 2009.

2000 Mathematics Subject Classification. Primary 65N30, 78A45, 35Q60.

Key words and phrases. Adaptivity, perfectly matched layer, a posteriori error analysis, cross gratings.

The first author's research was supported in part by the NSF grants DMS-0604790, CCF0514078, CCF-0830161, and EAR-0724527, the ONR grants N000140210365 and N000140910384, the National Science Foundation of China grant 10428105.

The second author's research was supported in part by the NSF EAR-0724656.

The third author's research was supported by the national basic research program under grant 2005CB321701, by the program for new century excellent talents in university of China, and by the NSF of China grant 10401016.

(C)2009 American Mathematical Society Reverts to public domain 28 years from publication 


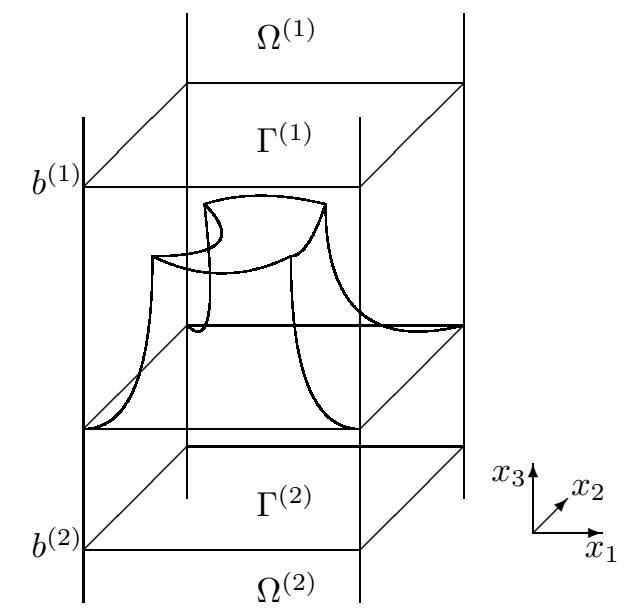

FiguRE 1. Geometry of the grating problem.

$\left.b^{(2)}<x_{3}<b^{(1)}\right\}$ that contains the structure, i.e., that there exist constants $\varepsilon^{(j)}, \mu^{(j)}, j=1,2$, such that

$$
\begin{aligned}
& \varepsilon(x)=\varepsilon^{(1)} I, \quad \mu(x)=\mu^{(1)} I, \quad \text { in } \Omega^{(1)}:=\left\{x: x_{3} \geq b^{(1)}\right\}, \\
& \varepsilon(x)=\varepsilon^{(2)} I, \quad \mu(x)=\mu^{(2)} I, \quad \text { in } \Omega^{(2)}:=\left\{x: x_{3} \leq b^{(2)}\right\},
\end{aligned}
$$

where $I$ is the $3 \times 3$ identity matrix. It is further assumed that $\varepsilon^{(1)}>0$ and $\mu^{(j)}>0, j=1,2$. However $\varepsilon^{(2)}$ may be complex according to the substrate material used in $\Omega^{(2)}$. Due to the periodic structure, the usual Sommerfeld or Silver-Müller radiation condition is no longer valid. Instead, the following radiation condition based on diffraction theory is employed: $(\mathbf{E}, \mathbf{H})$ is composed of bounded outgoing waves in $\Omega^{(1)}$ and $\Omega^{(2)}$ plus the incident wave $\left(\mathbf{E}_{\mathrm{I}}, \mathbf{H}_{\mathrm{I}}\right)$ in $\Omega^{(1)}$.

Scattering theory in biperiodic structures has many important applications in micro-optics, where the biperiodic structures are also termed as 2D gratings or crossed gratings. We refer to Dobson and Friedman [27, Abboud [1, Bao [7, Bao, Dobson, and Cox [11, Bao and Dobson [10, Bao and Zhou [13, Li [33, Yachin and Yasumoto [4], and Chang, Li, Chu, and Opsal 20] for the existence, uniqueness, and numerical approximations of solutions to $2 \mathrm{D}$ grating problems. An introduction to grating problems can be found in Petit 41. A more recent review on diffractive optics technology and its mathematical modeling can be found in Bao, Cowsar, and Masters [9.

Recently, we have developed an adaptive finite nodal element method combining with the PML technique (Chen and Wu [24] and Bao, Chen, and $\mathrm{Wu}[8]$ ) for 1D gratings (two-dimensional problems). The method can deal with extremely general diffractive structures and materials. It is stable, quickly convergent, and easy to implement by using existing FEM software for bounded domains. Moreover, the method produces the approximate electric field or magnetic field near the grating structure directly, which can be used in solving optimal design or inverse problems. There are two main difficulties in applying finite element methods to grating problems. The first is to truncate the open domain into a bounded computational domain. The second difficulty is concerned with resolving the singularity of the solutions. Usually, the grating surface is piecewise smooth and the dielectric coefficient 
$\varepsilon(x)$ is discontinuous across the surface. Thus the solution of (1.1) has singularities that slow down the finite element convergence when using uniform mesh refinements. In [24, 8], we applied a PML technique to truncate the unbounded domain and employed modern techniques of adaptive finite elements based on a posteriori error estimates to handle the second difficulty.

The PML technique, which was first proposed by Berenger [15, 16, is an important and popular mesh termination technique in computational wave propagation due to its effectiveness, simplicity, and flexibility [23, 25, 32, 45, 46]. The idea is to surround the computational domain by a nonphysical PML medium which has the remarkable property of being reflectionless for incident waves of any frequency or any incident direction, and waves decay exponentially in magnitude into the PML medium. In practical computations, the PML medium must be truncated and the truncation boundary generates reflected waves which can pollute the solution in the computational domain. We refer to [12, 18, 21] for convergence analyses of the PML problems for 3D Maxwell's equations.

A posteriori error estimates, which measure the actual discrete errors without the knowledge of limit solutions, are computable quantities in terms of the discrete solution. They are essential in designing algorithms for mesh modification that equidistribute the computational effort and optimize the computation. One of the main advantages of adaptivity is that it provides an effective approach for modeling multiscale phenomena 6, 8, 14, 22, 24, 35, 37, 48, 47. For the convergence of adaptive finite element methods, we refer to Dörfler 28, Morin, Nochetto, and Siebert [38, 39], Mekchay and Nochetto [34, and Chen and Dai [22]. For the quasi-optimality of adaptive finite element methods, we refer to Binev, Dahmen and DeVore [17, and Stevenson [4]. The adaptive FEM is attractive for grating problems whose solutions often have singularities due to the discontinuity of the dielectric coefficient. We refer to related work [21, 23, 19] for solving scattering problems in general media (nonperiodic) by using finite element methods together with the PML technique.

The purpose of this paper is to extend our previous work on 1D gratings $(24,8)$ to crossed gratings and to develop efficient numerical methods for solving the $2 \mathrm{D}$ grating problems. However, the techniques differ significantly because of the more complicated Maxwell's equations model in the case of 2D gratings.

We obtain the following results:

- Convergence of PML. Under assumptions for the uniqueness of the solution to the original scattering problem and on the PML medium parameter, we prove that the truncated PML problem attains a unique solution in $H$ (curl) and obtain an error estimate between the solution of the scattering problem and the solution of the truncated PML problem in the computational domain. The error estimate implies particularly that the PML solution converges exponentially to the scattering solution when either the PML medium parameter or the thickness of the layer is increased.

- A posteriori error estimates of edge element methods and an adaptive algorithm. To simplify the analysis and implementation, we first eliminate the nonhomogeneous Dirichlet boundary condition on the upper truncated boundary by subtracting the difference between the incident field and its mirror reflection in the PML medium to obtain a modified PML problem. Then we use Nédélec's edge element to discretize the modified PML 
problem and derive a a posteriori error estimate between the edge element solution and the original scattering solution in the computation domain. We emphasize that the a posteriori error estimate does not depend on the PML solution. One benefit of the modification is that, unlike our work for 1D grating [24, 8, we do not need the "exponential decay factors" for error indicators in the PML regions because the fields in the PML region already decay. The a posteriori error estimate, which consists of two parts, the PML error and the finite element discretization error, is used to design an adaptive algorithm to determine the PML parameters and choose elements for refinements. We present two numerical examples to illustrate the features of our adaptive algorithm.

The layout of the paper is as follows. In Section 2, the properties of the quasiperiodic functions in $H$ (curl) are discussed, a variational formulation for the $2 \mathrm{D}$ grating problem is introduced by using the transparent boundary condition, the PML formulation is presented, and existence, uniqueness, and convergence of the PML formulation are studied. Section 3 is devoted to the introduction of the edge element discretization. A crucial a posteriori error estimate is also stated. In Section 4, we prove the a posteriori error estimate, which offers the basis for the adaptive method. In Section 5, we discuss the implementation of the adaptive method and present two numerical examples to illustrate the competitive behavior of the method. The paper is completed with the proofs of several technical lemmas in the appendix.

\section{PML FORMULATION AND CONVERGENCE}

In this section, we introduce variational formulations for the 2D grating problem using the PML technique, which will be useful for subsequent edge element approximations, and the convergence analysis of the PML solution.

Let $\left(\mathbf{E}_{\mathrm{I}}, \mathbf{H}_{\mathrm{I}}\right)$ be the incoming plane waves that are incident upon the grating surface from the top:

$$
\mathbf{E}_{\mathrm{I}}=p e^{\mathrm{i} q \cdot x}, \quad \mathbf{H}_{\mathrm{I}}=s e^{\mathrm{i} q \cdot x}, \quad s=\frac{q \times p}{\omega \mu^{(1)}}, \quad p \cdot q=0,
$$

where $q=\left(\alpha_{1}, \alpha_{2},-\beta\right)^{\mathrm{T}}=\omega \sqrt{\varepsilon^{(1)} \mu^{(1)}}\left(\sin \theta_{1} \cos \theta_{2}, \sin \theta_{1} \sin \theta_{2},-\cos \theta_{1}\right)^{\mathrm{T}}$, and $\theta_{1}, \theta_{2}$ are incident angles satisfying $0 \leq \theta_{1}<\pi / 2,0 \leq \theta_{2}<2 \pi$. We are interested in quasi-periodic solutions, i.e., solutions $(\mathbf{E}, \mathbf{H})$ of (1.1) such that $\left(\mathbf{E}_{\alpha}, \mathbf{H}_{\alpha}\right)=$ $(\mathbf{E}, \mathbf{H}) e^{-\mathbf{i}\left(\alpha_{1} x_{1}+\alpha_{2} x_{2}\right)}$ are periodic in $x_{1}$ and $x_{2}$ with period $L_{1}>0$ and $L_{2}>0$, respectively.

2.1. Notation. In this subsection we introduce some notation and discuss the properties of quasi-periodic functions in $H$ (curl). For any cuboid domain

$$
G=\left\{0<x_{1}<L_{1}, 0<x_{2}<L_{2}, a<x_{3}<b\right\},
$$

denote by $L^{2}(G)$ the space of complex square integrable functions in $G$, and let

$$
H(\operatorname{curl}, G)=\left\{\varphi \in\left(L^{2}(G)\right)^{3}, \operatorname{curl} \varphi \in\left(L^{2}(G)\right)^{3}\right\}
$$

with the norm

$$
\|\varphi\|_{H(\operatorname{curl}, G)}=\left(\|\varphi\|_{\left(L^{2}(G)\right)^{3}}^{2}+\|\operatorname{curl} \varphi\|_{\left(L^{2}(G)\right)^{3}}^{2}\right)^{1 / 2} .
$$


Introduce the following periodic functional spaces:

$$
\begin{aligned}
& C_{\text {per }}^{\infty}(G)=\left\{f=\left.\tilde{f}\right|_{G}: \tilde{f} \in C^{\infty}\left(R^{3}\right),\right. \\
& \left.\tilde{f}\left(x_{1}+n_{1} L_{1}, x_{2}+n_{2} L_{2}, x_{3}\right)=\tilde{f}\left(x_{1}, x_{2}, x_{3}\right), \forall n_{1}, n_{2} \in Z\right\}, \\
& C_{0, \text { per }}^{\infty}(G)=\left\{f \in C_{\text {per }}^{\infty}(G): \operatorname{supp} f \subset\left\{a<x_{3}<b\right\}\right\}, \\
& \left.H_{\text {per }}(\operatorname{curl}, G)=\text { the closure of }\left(C_{\text {per }}^{\infty}(G)\right)^{3} \text { in } H \text { (curl, } G\right) \text {. }
\end{aligned}
$$

By using a similar argument to that for characterizing the space $H_{0}$ (curl) (cf. 31, Theorem 2.12] or [36, Theorem 3.33]) and using the convergence theory of Fourier series [29], we have the following lemma, which gives an alternative characterization of functions in $H_{\text {per }}(\operatorname{curl}, G)$. The proof is omitted to save space.

Lemma 2.1. Let $\nu_{1}=(1,0,0)^{\mathrm{T}}, \nu_{2}=(0,1,0)^{\mathrm{T}}$. Then

$$
\begin{aligned}
H_{\text {per }}(\operatorname{curl}, G)=\{\varphi \in H(\operatorname{curl}, G): & \varphi\left(0, x_{2}, x_{3}\right) \times \nu_{1}=\varphi\left(L_{1}, x_{2}, x_{3}\right) \times \nu_{1}, \\
& \left.\varphi\left(x_{1}, 0, x_{3}\right) \times \nu_{2}=\varphi\left(x_{1}, L_{2}, x_{3}\right) \times \nu_{2}\right\} .
\end{aligned}
$$

We remark that any $\varphi \in H_{\text {per }}(\operatorname{curl}, G)$ has a Fourier expansion which is convergent in the $H(\operatorname{curl}, G)$ norm:

$$
\varphi=\sum_{n \in Z^{2}} \hat{\varphi}_{n} e_{n}, \quad \text { where } e_{n}=e^{\mathrm{i}\left(\frac{2 \pi n_{1}}{L_{1}} x_{1}+\frac{2 \pi n_{2}}{L_{2}} x_{2}\right)} .
$$

Next we consider the quasi-periodic functions. Define

$$
H_{\mathrm{qper}}(\operatorname{curl}, G)=\left\{\varphi: e^{-\mathbf{i}\left(\alpha_{1} x_{1}+\alpha_{2} x_{2}\right)} \varphi \in H_{\mathrm{per}}(\operatorname{curl}, G)\right\} .
$$

From Lemma 2.1,

$$
\begin{aligned}
H_{\mathrm{qper}}(\operatorname{curl}, G)=\{\varphi \in H(\operatorname{curl}, G): & e^{\mathbf{i} \alpha_{1} L_{1}} \varphi\left(0, x_{2}, x_{3}\right) \times \nu_{1}=\varphi\left(L_{1}, x_{2}, x_{3}\right) \times \nu_{1}, \\
& \left.e^{\mathbf{i} \alpha_{2} L_{2}} \varphi\left(x_{1}, 0, x_{3}\right) \times \nu_{2}=\varphi\left(x_{1}, L_{2}, x_{3}\right) \times \nu_{2}\right\} .
\end{aligned}
$$

Let

$$
\Omega=\left\{x: 0<x_{1}<L_{1}, 0<x_{2}<L_{2} \text {, and } b^{(2)}<x_{3}<b^{(1)}\right\} .
$$

Denote by $\Gamma^{(j)}=\left\{x: 0<x_{1}<L_{1}, 0<x_{2}<L_{2}, x_{3}=b^{(j)}\right\}, j=1,2$, the upper and lower faces of $\Omega$. For any smooth vector field $\psi=\left(\psi_{1}, \psi_{2}, \psi_{3}\right)^{\mathrm{T}}$, denote by

$$
\psi_{\Gamma^{(j)}}=\left(\psi_{1}\left(x_{1}, x_{2}, b^{(j)}\right), \psi_{2}\left(x_{1}, x_{2}, b^{(j)}\right), 0\right)^{\mathrm{T}}
$$

its tangential component on the surface $\Gamma^{(j)}$. For any vector field $\varphi=\left(\varphi_{1}, \varphi_{2}, \varphi_{3}\right)^{\mathrm{T}}$ defined on $\Gamma^{(j)}, \operatorname{curl}_{\Gamma^{(j)}} \varphi=\frac{\partial \varphi_{2}}{\partial x_{1}}-\frac{\partial \varphi_{1}}{\partial x_{2}}$ is the surface scalar curl of the field $\varphi$. Introduce the following tangential functional spaces:

$$
\begin{aligned}
& T L^{2}\left(\Gamma^{(j)}\right)=\left\{\varphi \in\left(L^{2}\left(\Gamma^{(j)}\right)\right)^{3}, \varphi_{3}=0\right\}, \\
& T H_{\mathrm{qper}}^{-1 / 2}\left(\operatorname{curl}, \Gamma^{(j)}\right)=\left\{\varphi \in\left(H^{-1 / 2}\left(\Gamma^{(j)}\right)\right)^{3}, \operatorname{curl}_{\left.\Gamma^{(j)}\right)} \varphi \in H^{-1 / 2}\left(\Gamma^{(j)}\right), \varphi_{3}=0,\right. \\
& \quad e^{-\mathbf{i}\left(\alpha_{1} x_{1}+\alpha_{2} x_{2}\right)} \varphi_{m}, m=1,2 \text {, are periodic distributions } \\
& \text { with period } \left.L_{\ell} \text { in the } x_{\ell}(\ell=1,2) \text { direction }\right\} .
\end{aligned}
$$


We refer to 29] for the definition of periodic distributions. It is well known that any periodic distribution $f$ with period $L_{\ell}$ in the $x_{\ell}(\ell=1,2)$ direction has the following expansion [29, 42]:

$$
f=\sum_{n \in Z^{2}} b_{n} e^{\mathrm{i}\left(\frac{2 \pi n_{1}}{L_{1}} x_{1}+\frac{2 \pi n_{2}}{L_{2}} x_{2}\right)},
$$

where $\left\{b_{n}\right\}_{n \in Z^{2}}$ is a sequence of complex numbers satisfying

$$
\left|b_{n}\right| \leq c_{m}(1+|n|)^{m}, n \in Z^{2} \text {, for some } m=0,1,2, \ldots .
$$

Here $c_{m}$ is an appropriate positive number. Let

$$
\alpha_{j}^{n}=2 \pi n / L_{j}+\alpha_{j}, \quad j=1,2, \quad n \text { integer. }
$$

Then any quasi-periodic distribution $F$ with period $L_{\ell}$ in the $x_{\ell}(\ell=1,2)$ direction can be written

$$
F=\sum_{n=(l, m) \in Z^{2}} b_{n} e^{\mathrm{i}\left(\alpha_{1}^{l} x_{1}+\alpha_{2}^{m} x_{2}\right)},
$$

where $b_{n}$ satisfies (2.5).

For any quasi-periodic tangential vector field $\varphi$, which has the expansion

$$
\varphi=\sum_{l, m \in Z}\left(\varphi_{l, m, 1}, \varphi_{l, m, 2}, 0\right)^{\mathrm{T}} e^{\mathbf{i}\left(\alpha_{1}^{l} x_{1}+\alpha_{2}^{m} x_{2}\right)},
$$

its $T L^{2}\left(\Gamma^{(j)}\right)$ norm may be represented as

$$
\|\varphi\|_{T L^{2}\left(\Gamma^{(j)}\right)}^{2}=L_{1} L_{2} \sum_{l, m \in Z}\left(\left|\varphi_{l, m, 1}\right|^{2}+\left|\varphi_{l, m, 2}\right|^{2}\right) .
$$

Using series coefficients, the norm on the space $T H_{\mathrm{qper}}^{-1 / 2}\left(\operatorname{curl}, \Gamma^{(j)}\right)$ can be characterized by

$$
\begin{aligned}
\|\varphi\|_{T H_{\mathrm{qper}}^{-1 / 2}\left(\operatorname{curl}, \Gamma^{(j)}\right)}^{2}=L_{1} L_{2} & \sum_{l, m \in Z}\left(1+\left(\alpha_{1}^{l}\right)^{2}+\left(\alpha_{2}^{m}\right)^{2}\right)^{-1 / 2} \\
& \times\left[\left|\varphi_{l, m, 1}\right|^{2}+\left|\varphi_{l, m, 2}\right|^{2}+\left|\alpha_{1}^{l} \varphi_{l, m, 2}-\alpha_{2}^{m} \varphi_{l, m, 1}\right|^{2}\right] .
\end{aligned}
$$

The following trace regularity result for $H_{\mathrm{qper}}(\operatorname{curl}, \Omega)$ is useful in subsequent analysis.

Lemma 2.2. Let $\gamma_{0}=\max \left\{\sqrt{1+\left(b^{(1)}-b^{(2)}\right)^{-1}}, \sqrt{2}\right\}$. Then

$$
\left\|\psi_{\Gamma^{(j)}}\right\|_{T H_{\mathrm{qper}}^{-1 / 2}\left(\operatorname{curl}, \Gamma^{(j)}\right)} \leq \gamma_{0}\|\psi\|_{H(\operatorname{curl}, \Omega)} \quad \forall \psi \in H_{\mathrm{qper}}(\operatorname{curl}, \Omega) .
$$

Proof. First we have

$$
\begin{aligned}
\left(b^{(1)}-b^{(2)}\right)\left|\xi\left(b^{(j)}\right)\right|^{2} & =\int_{b^{(2)}}^{b^{(1)}}\left|\xi\left(x_{3}\right)\right|^{2} \mathrm{~d} x_{3}+\int_{b^{(2)}}^{b^{(1)}} \int_{x_{3}}^{b^{(j)}} \frac{\mathrm{d}}{\mathrm{d} \tau}|\xi(\tau)|^{2} \mathrm{~d} \tau \mathrm{d} x_{3} \\
& \leq \int_{b^{(2)}}^{b^{(1)}}\left|\xi\left(x_{3}\right)\right|^{2} \mathrm{~d} x_{3}+\left(b^{(1)}-b^{(2)}\right) \int_{b^{(2)}}^{b^{(1)}} 2\left|\xi\left(x_{3}\right)\right|\left|\xi^{\prime}\left(x_{3}\right)\right| \mathrm{d} x_{3},
\end{aligned}
$$

which implies by the Cauchy-Schwarz inequality that

$$
\frac{\left|\xi\left(b^{(j)}\right)\right|^{2}}{\left(1+\left(\alpha_{1}^{l}\right)^{2}+\left(\alpha_{2}^{m}\right)^{2}\right)^{1 / 2}} \leq\left(\gamma_{0}\right)^{2} \int_{b^{(2)}}^{b^{(1)}}\left|\xi\left(x_{3}\right)\right|^{2} \mathrm{~d} x_{3}+\frac{\int_{b^{(2)}}^{b^{(1)}}\left|\xi^{\prime}\left(x_{3}\right)\right|^{2} \mathrm{~d} x_{3}}{1+\left(\alpha_{1}^{l}\right)^{2}+\left(\alpha_{2}^{m}\right)^{2}}
$$


Since $\psi$ is quasi-periodic, it has the following expansion:

$$
\psi=\sum_{l, m \in Z}\left(\psi_{l, m, 1}\left(x_{3}\right), \psi_{l, m, 2}\left(x_{3}\right), \psi_{l, m, 3}\left(x_{3}\right)\right)^{\mathrm{T}} e^{\mathbf{i}\left(\alpha_{1}^{l} x_{1}+\alpha_{2}^{m} x_{2}\right)} .
$$

A simple calculation yields that

$$
\begin{aligned}
\|\psi\|_{H(\mathrm{curl}, \Omega)}^{2}= & L_{1} L_{2} \sum_{l, m \in Z} \int_{b^{(2)}}^{b^{(1)}}\left(\left|\psi_{l, m, 1}\left(x_{3}\right)\right|^{2}+\left|\psi_{l, m, 2}\left(x_{3}\right)\right|^{2}+\left|\psi_{l, m, 3}\left(x_{3}\right)\right|^{2}\right. \\
& +\left|\mathbf{i} \alpha_{2}^{m} \psi_{l, m, 3}\left(x_{3}\right)-\psi_{l, m, 2}^{\prime}\left(x_{3}\right)\right|^{2}+\left|\psi_{l, m, 1}^{\prime}\left(x_{3}\right)-\mathbf{i} \alpha_{1}^{l} \psi_{l, m, 3}\left(x_{3}\right)\right|^{2} \\
& \left.+\left|\alpha_{1}^{l} \psi_{l, m, 2}\left(x_{3}\right)-\alpha_{2}^{m} \psi_{l, m, 1}\left(x_{3}\right)\right|^{2}\right) \mathrm{d} x_{3} .
\end{aligned}
$$

From the definition (2.7),

$$
\begin{aligned}
& \left\|\psi_{\Gamma^{(j)}}\right\|_{T H_{\mathrm{qper}}^{-1 / 2}\left(\operatorname{curl}, \Gamma^{(j)}\right)}^{2}= \\
& \quad L_{1} L_{2} \sum_{l, m \in Z} \frac{\left|\psi_{l, m, 1}\left(b^{(j)}\right)\right|^{2}+\left|\psi_{l, m, 2}\left(b^{(j)}\right)\right|^{2}+\left|\alpha_{1}^{l} \psi_{l, m, 2}\left(b^{(j)}\right)-\alpha_{2}^{m} \psi_{l, m, 1}\left(b^{(j)}\right)\right|^{2}}{\left(1+\left(\alpha_{1}^{l}\right)^{2}+\left(\alpha_{2}^{m}\right)^{2}\right)^{1 / 2}} .
\end{aligned}
$$

Moreover, it follows from (2.8) that

$$
\begin{aligned}
& \frac{\left|\psi_{l, m, 1}\left(b^{(j)}\right)\right|^{2}+\left|\psi_{l, m, 2}\left(b^{(j)}\right)\right|^{2}+\left|\alpha_{1}^{l} \psi_{l, m, 2}\left(b^{(j)}\right)-\alpha_{2}^{m} \psi_{l, m, 1}\left(b^{(j)}\right)\right|^{2}}{\left(1+\left(\alpha_{1}^{l}\right)^{2}+\left(\alpha_{2}^{m}\right)^{2}\right)^{1 / 2}} \\
& \leq \int_{b^{(2)}}^{b^{(1)}}\left\{\left(\gamma_{0}\right)^{2}\left(\left|\psi_{l, m, 1}\left(x_{3}\right)\right|^{2}+\left|\psi_{l, m, 2}\left(x_{3}\right)\right|^{2}+\left|\alpha_{1}^{l} \psi_{l, m, 2}\left(x_{3}\right)-\alpha_{2}^{m} \psi_{l, m, 1}\left(x_{3}\right)\right|^{2}\right)\right. \\
& \quad+\left(1+\left(\alpha_{1}^{l}\right)^{2}+\left(\alpha_{2}^{m}\right)^{2}\right)^{-1}\left(2\left|\psi_{l, m, 1}^{\prime}\left(x_{3}\right)-\mathbf{i} \alpha_{1}^{l} \psi_{l, m, 3}\left(x_{3}\right)\right|^{2}\right. \\
& \quad+2\left(\alpha_{1}^{l}\right)^{2}\left|\psi_{l, m, 3}\left(x_{3}\right)\right|^{2}+2\left|\mathbf{i} \alpha_{2}^{m} \psi_{l, m, 3}\left(x_{3}\right)-\psi_{l, m, 2}^{\prime}\left(x_{3}\right)\right|^{2}+2\left(\alpha_{2}^{m}\right)^{2}\left|\psi_{l, m, 3}\left(x_{3}\right)\right|^{2} \\
& \left.\left.\quad+\left|\alpha_{1}^{l} \psi_{l, m, 2}^{\prime}\left(x_{3}\right)-\mathbf{i} \alpha_{1}^{l} \alpha_{2}^{m} \psi_{l, m, 3}\left(x_{3}\right)+\mathbf{i} \alpha_{1}^{l} \alpha_{2}^{m} \psi_{l, m, 3}\left(x_{3}\right)-\alpha_{2}^{m} \psi_{l, m, 1}^{\prime}\left(x_{3}\right)\right|^{2}\right)\right\} \mathrm{d} x_{3} \\
& \leq \int_{b^{(2)}}^{b^{(1)}}\left\{\left(\gamma_{0}\right)^{2}\left(\left|\psi_{l, m, 1}\left(x_{3}\right)\right|^{2}+\left|\psi_{l, m, 2}\left(x_{3}\right)\right|^{2}+\left|\alpha_{1}^{l} \psi_{l, m, 2}\left(x_{3}\right)-\alpha_{2}^{m} \psi_{l, m, 1}\left(x_{3}\right)\right|^{2}\right)\right. \\
& +2\left(1+\left(\alpha_{1}^{l}\right)^{2}+\left(\alpha_{2}^{m}\right)^{2}\right)^{-1}\left(\left(1+\left(\alpha_{2}^{m}\right)^{2}\right)\left|\psi_{l, m, 1}^{\prime}\left(x_{3}\right)-\mathbf{i} \alpha_{1}^{l} \psi_{l, m, 3}\left(x_{3}\right)\right|^{2}\right. \\
& \left.\left.+\left(1+\left(\alpha_{1}^{l}\right)^{2}\right)\left|\mathbf{i} \alpha_{2}^{m} \psi_{l, m, 3}\left(x_{3}\right)-\psi_{l, m, 2}^{\prime}\left(x_{3}\right)\right|^{2}+\left(\left(\alpha_{1}^{l}\right)^{2}+\left(\alpha_{2}^{m}\right)^{2}\right)\left|\psi_{l, m, 3}\left(x_{3}\right)\right|^{2}\right)\right\} \mathrm{d} x_{3},
\end{aligned}
$$

which implies, together with (2.9), that Lemma 2.2 holds.

2.2. A variational formulation of the grating problem. Based on the capacity operator [3, we introduce the variational formulation with transparent boundary condition, which serves as the basis of the analysis in this paper.

We wish to reduce the problem to the bounded domain $\Omega$. The radiation condition for the diffraction problem insists that $(\mathbf{E}, \mathbf{H})$ is composed of bounded outgoing plane waves in $\Omega^{(1)}$ and $\Omega^{(2)}$, plus the incident wave $\left(\mathbf{E}_{\mathrm{I}}, \mathbf{H}_{\mathrm{I}}\right)$ in $\Omega^{(1)}$. Denote by

$$
\mathbf{E}_{\mathrm{I}}^{(1)}=\mathbf{E}_{\mathrm{I}}, \quad \mathbf{H}_{\mathrm{I}}^{(1)}=\mathbf{H}_{\mathrm{I}}, \quad \mathbf{E}_{\mathrm{I}}^{(2)}=\mathbf{H}_{\mathrm{I}}^{(2)}=0 .
$$

Since $\left(\mathbf{E}_{\alpha}, \mathbf{H}_{\alpha}\right)=(\mathbf{E}, \mathbf{H}) e^{-\mathbf{i}\left(\alpha_{1} x_{1}+\alpha_{2} x_{2}\right)}$ is periodic, it follows from the Fourier series expansion of $\left(\mathbf{E}_{\alpha}, \mathbf{H}_{\alpha}\right)$ and the method of separation of variables that $(\mathbf{E}, \mathbf{H})$ 
has the following Rayleigh expansion in $\Omega^{(1)}$ and $\Omega^{(2)}$ :

$$
\begin{aligned}
\mathbf{E}-\mathbf{E}_{\mathrm{I}}^{(j)} & =\sum_{l, m \in Z} p_{l, m}^{(j)} e^{\mathbf{i} l_{l, m}^{(j)} \cdot x}, \\
\mathbf{H}-\mathbf{H}_{\mathrm{I}}^{(j)} & =\sum_{l, m \in Z} s_{l, m}^{(j)} e^{\mathbf{i} q_{l, m}^{(j)} \cdot x}, \quad x \in \Omega^{(j)}, j=1,2,
\end{aligned}
$$

where

$$
\begin{gathered}
s_{l, m}^{(j)}=\frac{1}{\omega \mu^{(j)}} q_{l, m}^{(j)} \times p_{l, m}^{(j)}, \quad p_{l, m}^{(j)} \cdot q_{l, m}^{(j)}=0, \\
q_{l, m}^{(j)}=\left(\alpha_{1}^{l}, \alpha_{2}^{m},(-1)^{j-1} \beta_{l, m}^{(j)}\right)^{\mathrm{T}},
\end{gathered}
$$

and $\beta_{l, m}^{(j)}$ satisfy:

$$
\left(\beta_{l, m}^{(j)}\right)^{2}=\omega^{2} \varepsilon^{(j)} \mu^{(j)}-\left(\alpha_{1}^{l}\right)^{2}-\left(\alpha_{2}^{m}\right)^{2}, \quad \operatorname{Im} \beta_{l, m}^{(j)} \geq 0, \quad j=1,2 .
$$

Note that $\beta_{0,0}^{(1)}=\beta$ by definition. Here, we exclude resonances by assuming that

$$
\omega^{2} \varepsilon^{(j)} \mu^{(j)} \neq\left(\alpha_{1}^{l}\right)^{2}+\left(\alpha_{2}^{m}\right)^{2} \quad \text { for all } l, m \in Z, j=1,2 .
$$

Let $\nu$ stand for the unit outer normal to $\partial \Omega$. Noting that $\nu=\left(0,0,(-1)^{j-1}\right)$ on $\Gamma^{(j)}$, it follows from (2.11)-(2.13) that

$$
\begin{aligned}
s_{l, m}^{(j)} \times\left.\nu\right|_{\Gamma^{(j)}}=\frac{1}{\omega \mu^{(j)} \beta_{l, m}^{(j)}}\left(\omega^{2} \varepsilon^{(j)} \mu^{(j)} p_{l, m, 1}^{(j)}+\alpha_{2}^{m}\left(\alpha_{1}^{l} p_{l, m, 2}^{(j)}-\alpha_{2}^{m} p_{l, m, 1}^{(j)}\right),\right. \\
\left.\omega^{2} \varepsilon^{(j)} \mu^{(j)} p_{l, m, 2}^{(j)}-\alpha_{1}^{l}\left(\alpha_{1}^{l} p_{l, m, 2}^{(j)}-\alpha_{2}^{m} p_{l, m, 1}^{(j)}\right), 0\right)^{\mathrm{T}},
\end{aligned}
$$

where $p_{l, m, 1}^{(j)}$ and $p_{l, m, 2}^{(j)}$ are the first and second components of the vector $p_{l, m}^{(j)}$, respectively.

For any tangential vector field $\varphi \in T H_{\mathrm{qper}}^{-1 / 2}\left(\operatorname{curl}, \Gamma^{(j)}\right)$ which has the expansion

$$
\varphi=\sum_{l, m \in Z}\left(\varphi_{l, m, 1}^{(j)}, \varphi_{l, m, 2}^{(j)}, 0\right)^{\mathrm{T}} e^{\mathbf{i}\left(\alpha_{1}^{l} x_{1}+\alpha_{2}^{m} x_{2}\right)}
$$

define the following capacity operator $T^{(j)}$ :

$$
T^{(j)} \varphi=\sum_{l, m \in Z}\left(r_{l, m, 1}^{(j)}, r_{l, m, 2}^{(j)}, 0\right)^{\mathrm{T}} e^{\mathbf{i}\left(\alpha_{1}^{l} x_{1}+\alpha_{2}^{m} x_{2}\right)}, \quad j=1,2,
$$

where

$$
\begin{aligned}
r_{l, m, 1}^{(j)} & =\frac{\omega^{2} \varepsilon^{(j)} \mu^{(j)} \varphi_{l, m, 1}^{(j)}+\alpha_{2}^{m}\left(\alpha_{1}^{l} \varphi_{l, m, 2}^{(j)}-\alpha_{2}^{m} \varphi_{l, m, 1}^{(j)}\right)}{\omega \mu^{(j)} \beta_{l, m}^{(j)}}, \\
r_{l, m, 2}^{(j)} & =\frac{\omega^{2} \varepsilon^{(j)} \mu^{(j)} \varphi_{l, m, 2}^{(j)}-\alpha_{1}^{l}\left(\alpha_{1}^{l} \varphi_{l, m, 2}^{(j)}-\alpha_{2}^{m} \varphi_{l, m, 1}^{(j)}\right)}{\omega \mu^{(j)} \beta_{l, m}^{(j)}} .
\end{aligned}
$$

With this notation in mind, it follows from (2.14) and the Rayleigh expansion $(\mathbf{E}, \mathbf{H})$ in $\Omega^{(j)}, j=1,2$, defined in (2.10), that the following transparent boundary conditions hold:

$$
\left(\mathbf{H}-\mathbf{H}_{\mathrm{I}}\right) \times \nu=T^{(1)}\left(\mathbf{E}-\mathbf{E}_{\mathrm{I}}\right)_{\Gamma^{(1)}} \quad \text { on } \quad \Gamma^{(1)}, \quad \mathbf{H} \times \nu=T^{(2)} \mathbf{E}_{\Gamma^{(2)}} \quad \text { on } \quad \Gamma^{(2)} .
$$


We next present a variational formulation of the Maxwell system (1.1) in the space $H_{\text {qper }}(\operatorname{curl}, \Omega)$. By eliminating the magnetic field $\mathbf{H}$ from (1.1), we obtain

$$
\operatorname{curl} \mu^{-1} \operatorname{curl} \mathbf{E}-\omega^{2} \varepsilon \mathbf{E}=0 \text { in } \Omega .
$$

Multiplying the complex conjugate of a test function $\psi$ in $H_{\mathrm{qper}}(\mathrm{curl}, \Omega)$, integrating over $\Omega$, and using integration by parts, we arrive at the variational form for the scattering problem (1.1): find $\mathbf{E} \in H_{\mathrm{qper}}(\operatorname{curl}, \Omega)$ such that

$$
A(\mathbf{E}, \psi)=\left\langle f_{\mathrm{I}}, \psi\right\rangle \quad \forall \psi \in H_{\text {qper }}(\operatorname{curl}, \Omega),
$$

where the sesquilinear form

$$
A(\varphi, \psi)=\int_{\Omega} \mu^{-1} \operatorname{curl} \varphi \cdot \operatorname{curl} \bar{\psi}-\int_{\Omega} \omega^{2} \varepsilon \varphi \cdot \bar{\psi}-\mathbf{i} \omega \sum_{j=1}^{2} \int_{\Gamma^{(j)}} T^{(j)} \varphi_{\Gamma^{(j)}} \cdot \bar{\psi}_{\Gamma^{(j)}}
$$

and

$$
\begin{aligned}
\left\langle f_{\mathrm{I}}, \psi\right\rangle & =\mathbf{i} \omega \int_{\Gamma^{(1)}}\left(\mathbf{H}_{\mathrm{I}} \times \nu-T^{(1)}\left(\mathbf{E}_{\mathrm{I}}\right)_{\Gamma^{(1)}}\right) \cdot \bar{\psi}_{\Gamma^{(1)}} \\
& =-2 \mathbf{i} \omega \int_{\Gamma^{(1)}}\left(T^{(1)}\left(\mathbf{E}_{\mathrm{I}}\right)_{\Gamma^{(1)}}\right) \cdot \bar{\psi}_{\Gamma^{(1)}} .
\end{aligned}
$$

Here we have used the identity

$$
\mathbf{H}_{\mathrm{I}} \times \nu=-T^{(1)}\left(\mathbf{E}_{\mathrm{I}}\right)_{\Gamma^{(1)}} \text { on } \Gamma^{(1)} .
$$

Throughout the paper, it is assumed that the variational problem (2.19) attains a unique weak solution in $H_{\text {qper }}(\operatorname{curl}, \Omega)$. Then the general theory in Babuška and Aziz [5, Chap. 5] implies that there exists a constant $\gamma_{1}>0$ such that the following inf-sup condition holds:

$$
\sup _{0 \neq \psi \in H_{\mathrm{qper}(\operatorname{curl}, \Omega)}} \frac{|A(\varphi, \psi)|}{\|\psi\|_{H(\operatorname{curl}, \Omega)}} \geq \gamma_{1}\|\varphi\|_{H(\operatorname{curl}, \Omega)} \quad \forall \varphi \in H_{\mathrm{qper}}(\operatorname{curl}, \Omega) .
$$

See Bao and Dobson [10 and Ammari and Bao 2] for additional discussions on the solvability of the variational problem in the isotropic case. The questions on existence and uniqueness in the present general case are open.

2.3. PML formulation. Now we turn to the introduction of absorbing PML layers. The computational domain $\Omega$ is surrounded with two PML layers of thickness $\delta^{(1)}$ and $\delta^{(2)}$ in $\Omega^{(1)}$ and $\Omega^{(2)}$, respectively. The specially designed model medium in the PML layers should basically be chosen so that either the wave never reaches its external boundary or the amplitude of the reflected wave is so small that it does not essentially contaminate the solution in $\Omega$. Let $s(\tau)=s_{1}(\tau)+\mathbf{i} s_{2}(\tau)$ be the model medium property which is continuous and satisfies

(2.24) $s_{1}=1, s_{2}=0$, if $b^{(2)} \leq \tau \leq b^{(1)}$, and $s_{1} \geq 1, s_{2}>0$, otherwise.

We remark that, in contrast to the original PML condition which takes $s_{1} \equiv 1$ in the PML region, a variable $s_{1}$ is allowed in order to attenuate both the outgoing and evanescent waves there. The advantage of this extension makes our method insensitive to the distance of the PML region from the structure. Following the general idea in designing PML absorbing layers [30, 45], we introduce the PML by complex coordinate stretching:

$$
\hat{x}_{3}=\int_{0}^{x_{3}} s(\tau) \mathrm{d} \tau .
$$


Let

$$
\hat{x}=\left(x_{1}, x_{2}, \hat{x}_{3}\right)^{\mathrm{T}} .
$$

It is clear that $\hat{x}_{3}=x_{3}$ for $x \in \Omega$ and

$$
\operatorname{curl} \operatorname{diag}\left(1,1, s\left(x_{3}\right)\right) \varphi=\operatorname{diag}\left(s\left(x_{3}\right), s\left(x_{3}\right), 1\right) \operatorname{curl}_{\hat{x}} \varphi .
$$

Introduce the new field $(\widehat{\mathbf{E}}, \widehat{\mathbf{H}})$ :

$$
\begin{aligned}
\widehat{\mathbf{E}}(x) & = \begin{cases}\mathbf{E}_{\mathrm{I}}^{(j)}(x)+\operatorname{diag}\left(1,1, s\left(x_{3}\right)\right)\left(\mathbf{E}(\hat{x})-\mathbf{E}_{\mathrm{I}}^{(j)}(\hat{x})\right), & \text { if } x \in \Omega^{(j)}, \\
\mathbf{E}(x), & \text { if } x \in \Omega ;\end{cases} \\
\widehat{\mathbf{H}}(x) & = \begin{cases}\mathbf{H}_{\mathrm{I}}^{(j)}(x)+\operatorname{diag}\left(1,1, s\left(x_{3}\right)\right)\left(\mathbf{H}(\hat{x})-\mathbf{H}_{\mathrm{I}}^{(j)}(\hat{x})\right), & \text { if } x \in \Omega^{(j)}, \\
\mathbf{H}(x), & \text { if } x \in \Omega .\end{cases}
\end{aligned}
$$

It follows from (1.1) and (2.25) that the field $(\widehat{\mathbf{E}}, \widehat{\mathbf{H}})$ satisfies the following Maxwelltype equations:

$$
\begin{array}{ll}
\operatorname{curl}\left(\widehat{\mathbf{E}}-\mathbf{E}_{\mathrm{I}}(x)\right)=\mathbf{i} \omega \hat{\mu}\left(\widehat{\mathbf{H}}-\mathbf{H}_{\mathrm{I}}(x)\right), & \\
\operatorname{curl}\left(\widehat{\mathbf{H}}-\mathbf{H}_{\mathrm{I}}(x)\right)=-\mathbf{i} \omega \hat{\varepsilon}\left(\widehat{\mathbf{E}}-\mathbf{E}_{\mathrm{I}}(x)\right), & \text { if } x \in \Omega^{(1)}, \\
\operatorname{curl} \widehat{\mathbf{E}}=\mathbf{i} \omega \hat{\mu} \widehat{\mathbf{H}}, \quad \operatorname{curl}_{x} \widehat{\mathbf{H}}=-\mathbf{i} \omega \hat{\varepsilon} \widehat{\mathbf{E}}, & \text { otherwise. }
\end{array}
$$

where

$$
\hat{\varepsilon}=\operatorname{diag}\left(s\left(x_{3}\right), s\left(x_{3}\right), 1 / s\left(x_{3}\right)\right) \varepsilon, \quad \hat{\mu}=\operatorname{diag}\left(s\left(x_{3}\right), s\left(x_{3}\right), 1 / s\left(x_{3}\right)\right) \mu .
$$

Define the PML regions

$$
\begin{aligned}
& \Omega^{(1, \mathrm{PML})}=\left\{x: 0<x_{1}<L_{1}, 0<x_{2}<L_{2} \text { and } b^{(1)}<x_{3}<b^{(1)}+\delta^{(1)}\right\}, \\
& \Omega^{(2, \mathrm{PML})}=\left\{x: 0<x_{1}<L_{1}, 0<x_{2}<L_{2} \text { and } b^{(2)}-\delta^{(2)}<x_{3}<b^{(2)}\right\} .
\end{aligned}
$$

It is easy to see from (2.26) and the Rayleigh expansion (2.10) that the outgoing waves $\left(\widehat{\mathbf{E}}(x)-\mathbf{E}_{\mathrm{I}}(x), \widehat{\mathbf{H}}(x)-\mathbf{H}_{\mathrm{I}}(x)\right)$ in $\Omega^{(1)}$ and $(\widehat{\mathbf{E}}(x), \widehat{\mathbf{H}}(x))$ in $\Omega^{(2)}$ decay exponentially as $\left|x_{3}\right| \rightarrow \infty$. The perfect conductor boundary condition can thus be imposed on

$$
\begin{aligned}
& \Gamma^{(1, \mathrm{PML})}=\left\{x: 0<x_{1}<L_{1}, 0<x_{2}<L_{2}, x_{3}=b^{(1)}+\delta^{(1)}\right\}, \\
& \Gamma^{(2, \mathrm{PML})}=\left\{x: 0<x_{1}<L_{1}, 0<x_{2}<L_{2}, x_{3}=b^{(2)}-\delta^{(2)}\right\}
\end{aligned}
$$

to truncate the PML media. We arrive at the following truncated PML problem: Find a quasi-periodic field $\left(\mathbf{E}^{\mathrm{PML}}(x), \mathbf{H}^{\mathrm{PML}}(x)\right)$ such that

$$
\begin{cases}\operatorname{curl} \mathbf{E}^{\mathrm{PML}}-\mathbf{i} \omega \hat{\mu} \mathbf{H}^{\mathrm{PML}}=f_{H}, & \operatorname{curl} \mathbf{H}^{\mathrm{PML}}+\mathbf{i} \omega \hat{\varepsilon} \mathbf{E}^{\mathrm{PML}}=f_{E}, \\ \mathbf{E}^{\mathrm{PML}} \times \nu=\mathbf{E}_{\mathrm{I}}(x) \times \nu \text { on } \Gamma^{(1, \mathrm{PML})}, & \mathbf{E}^{\mathrm{PML}} \times \nu=0 \text { on } \Gamma^{(2, \mathrm{PML})},\end{cases}
$$

where

$$
\left\{\begin{array}{lc}
f_{H}=\operatorname{curl} \mathbf{E}_{\mathrm{I}}(x)-\mathbf{i} \omega \hat{\mu} \mathbf{H}_{\mathrm{I}}(x), f_{E}=\operatorname{curl} \mathbf{H}_{\mathrm{I}}(x)+\mathbf{i} \omega \hat{\varepsilon} \mathbf{E}_{\mathrm{I}}(x), & \text { if } x \in \Omega^{(1, \mathrm{PML})} \\
f_{H}=f_{E}=0, & \text { otherwise. }
\end{array}\right.
$$

We next present a weak formulation of the PML problem (2.29) in

$$
D=\left\{x: 0<x_{1}<L_{1}, 0<x_{2}<L_{2}, b^{(2)}-\delta^{(2)}<x_{3}<b^{(1)}+\delta^{(1)}\right\} .
$$

Eliminating the magnetic field $\mathbf{H}^{\mathrm{PML}}$ from (2.29) yields

$$
\operatorname{curl}\left(\hat{\mu}^{-1} \operatorname{curl} \mathbf{E}^{\mathrm{PML}}\right)-\omega^{2} \hat{\varepsilon} \mathbf{E}^{\mathrm{PML}}=g \quad \text { in } D,
$$


where

$$
g(x)= \begin{cases}\operatorname{curl}\left(\hat{\mu}^{-1} \operatorname{curl} \mathbf{E}_{\mathrm{I}}(x)\right)-\omega^{2} \hat{\varepsilon} \mathbf{E}_{\mathrm{I}}(x), & \text { if } x \in \Omega^{(1, \mathrm{PML})} \\ 0, & \text { otherwise. }\end{cases}
$$

For $G=D, \Omega, \Omega^{(1, \mathrm{PML})}$, or $\Omega^{(2, \mathrm{PML})}$, introduce the sesquilinear form $a_{G}: H_{\mathrm{qper}}(\operatorname{curl}$, $G) \times H_{\text {qper }}(\operatorname{curl}, G) \rightarrow \mathbf{C}$ as

$$
a_{G}(\varphi, \psi)=\int_{G} \hat{\mu}^{-1} \operatorname{curl} \varphi \cdot \operatorname{curl} \bar{\psi}-\int_{G} \omega^{2} \hat{\varepsilon} \varphi \cdot \bar{\psi}
$$

Define $\stackrel{\circ}{H}_{\text {qper }}(\operatorname{curl}, D)=\left\{\varphi \in H_{\mathrm{qper}}(\operatorname{curl}, D), \varphi \times \nu=0\right.$ on $\left.\Gamma^{(j, \mathrm{PML})}, j=1,2\right\}$. The weak formulation of the PML model (2.29) reads as follows: find $\mathbf{E}^{\mathrm{PML}} \in$ $H_{\text {qper }}(\operatorname{curl}, D)$, such that $\mathbf{E}^{\mathrm{PML}} \times \nu=\mathbf{E}_{\mathrm{I}} \times \nu$ on $\Gamma^{(1, \mathrm{PML})}, \mathbf{E}^{\mathrm{PML}} \times \nu=0$ on $\Gamma^{(2, \mathrm{PML})}$, and

$$
a_{D}\left(\mathbf{E}^{\mathrm{PML}}, \psi\right)=\int_{D} g \cdot \bar{\psi}, \quad \forall \psi \in \stackrel{\circ}{H}_{\mathrm{qper}}(\operatorname{curl}, D) .
$$

We discuss the existence and convergence of the above weak formulation in the next subsection, and introduce its edge element discretization in Section 3.

2.4. Convergence of the PML solution. Our goal is to prove the existence and uniqueness of the solution of the above PML problem (2.34) and derive an error estimate between $\mathbf{E}^{\mathrm{PML}}$ and $\mathbf{E}$, the solution of the original $2 \mathrm{D}$ grating problem (2.19) in $\Omega$. To achieve this goal, we first find an equivalent formulation of (2.34) in the domain $\Omega$. For any vector $v=\left(v_{1}, v_{2}, v_{3}\right)^{\mathrm{T}}$, denote $v^{\prime}=\left(v_{1}, v_{2},-v_{3}\right)^{\mathrm{T}}$. Similar to the Rayleigh expansion (2.10), it deduces from (2.29) and (2.30) that, for $x \in \Omega^{(j, \mathrm{PML})}, j=1,2$,

$$
\begin{gathered}
\mathbf{E}^{\mathrm{PML}}(x)-\mathbf{E}_{\mathrm{I}}^{(j)}(x)=\operatorname{diag}\left(1,1, s\left(x_{3}\right)\right) \sum_{l, m \in Z}\left(p_{l, m}^{(j)} e^{\mathbf{i} q_{l, m}^{(j)} \cdot \hat{x}}-\zeta_{l, m}^{(j)}\left(p_{l, m}^{(j)}\right)^{\prime} e^{\mathbf{i}\left(q_{l, m}^{(j)}\right)^{\prime} \cdot \hat{x}}\right)^{\mathrm{T}}, \\
\mathbf{H}^{\mathrm{PML}}(x)-\mathbf{H}_{\mathrm{I}}^{(j)}(x)=\operatorname{diag}\left(1,1, s\left(x_{3}\right)\right) \sum_{l, m \in Z}\left(s_{l, m}^{(j)} e^{\mathbf{i} q_{l, m}^{(j)} \cdot \hat{x}}+\zeta_{l, m}^{(j)}\left(s_{l, m}^{(j)}\right)^{\prime} e^{\mathbf{i}\left(q_{l, m}^{(j)}\right)^{\prime} \cdot \hat{x}}\right)^{\mathrm{T}},
\end{gathered}
$$

where $q_{l, m}^{(j)}$ is defined in (2.12), $p_{l, m}^{(j)}$ and $s_{l, m}^{(j)}$ satisfy

$$
s_{l, m}^{(j)}=\frac{1}{\omega \mu^{(j)}} q_{l, m}^{(j)} \times p_{l, m}^{(j)}, \quad p_{l, m}^{(j)} \cdot q_{l, m}^{(j)}=0,
$$

and

$$
\zeta_{l, m}^{(1)}=e^{2 \mathbf{i} \beta_{l, m}^{(1)} \int_{0}^{b^{(1)}+\delta(1)} s(\tau) d \tau}, \quad \zeta_{l, m}^{(2)}=e^{2 \mathbf{i} \beta_{l, m}^{(2)} \int_{b(2)}^{0} \delta^{(2)} s(\tau) d \tau} .
$$

It is clear that

$$
\begin{aligned}
\left(\mathbf{E}^{\mathrm{PML}}(x)\right. & \left.-\mathbf{E}_{\mathrm{I}}^{(j)}(x)\right)_{\Gamma^{(j)}} \\
= & \sum_{l, m \in Z}\left(p_{l, m, 1}^{(j)}, p_{l, m, 2}^{(j)}, 0\right)^{\mathrm{T}} e^{\mathbf{i} q_{l, m}^{(j)} \cdot x}\left(1-\zeta_{l, m}^{(j)} e^{(-1)^{j} 2 \mathbf{i} \beta_{l, m}^{(j)} b^{(j)}}\right), \\
\left(\mathbf{H}^{\mathrm{PML}}(x)\right. & \left.-\mathbf{H}_{\mathrm{I}}^{(j)}(x)\right) \times \nu \\
& =\sum_{l, m \in Z}\left(s_{l, m}^{(j)} \times \nu\right) e^{\mathbf{i} q_{l, m}^{(j)} \cdot x}\left(1+\zeta_{l, m}^{(j)} e^{(-1)^{j} 2 \mathbf{i} \beta_{l, m}^{(j)} b^{(j)}}\right) \text { on } \Gamma^{(j)} .
\end{aligned}
$$


For any tangential vector field $\varphi \in T H_{\mathrm{qper}}^{-1 / 2}\left(\operatorname{curl}, \Gamma^{(j)}\right)$ which has the expansion

$$
\varphi=\sum_{l, m \in Z}\left(\varphi_{l, m, 1}^{(j)}, \varphi_{l, m, 2}^{(j)}, 0\right)^{\mathrm{T}} e^{\mathbf{i}\left(\alpha_{1}^{l} x_{1}+\alpha_{2}^{m} x_{2}\right)}
$$

define the following capacity operator $T^{(j, \mathrm{PML})}$ :

$$
T^{(j, \mathrm{PML})} \varphi=\sum_{l, m \in Z}\left(r_{l, m, 1}^{(j)}, r_{l, m, 2}^{(j)}, 0\right)^{\mathrm{T}} \operatorname{coth}\left(-\mathbf{i} \beta_{l, m}^{(j)} \sigma^{(j)}\right) e^{\mathbf{i}\left(\alpha_{1}^{l} x_{1}+\alpha_{2}^{m} x_{2}\right)}, j=1,2
$$

where $\operatorname{coth}(\tau)=\frac{e^{\tau}+e^{-\tau}}{e^{\tau}-e^{-\tau}}, r_{l, m, 1}^{(j)}$ and $r_{l, m, 2}^{(j)}$ are defined in (2.16), and

$$
\sigma^{(1)}=\int_{b^{(1)}}^{b^{(1)}+\delta^{(1)}} s(\tau) \mathrm{d} \tau, \quad \sigma^{(2)}=\int_{b^{(2)}-\delta^{(2)}}^{b^{(2)}} s(\tau) \mathrm{d} \tau .
$$

Since $\left(1+\zeta_{l, m}^{(j)} e^{(-1)^{j} 2 \mathbf{i} \beta_{l, m}^{(j)} b^{(j)}}\right) /\left(1-\zeta_{l, m}^{(j)} e^{(-1)^{j} 2 \mathbf{i} \beta_{l, m}^{(j)} b^{(j)}}\right)=\operatorname{coth}\left(-\mathbf{i} \beta_{l, m}^{(j)} \sigma^{(j)}\right)$, it follows from (2.37) $-(2.39)$ that

$$
\begin{aligned}
\left(\mathbf{H}^{\mathrm{PML}}-\mathbf{H}_{\mathrm{I}}\right) & \times \nu=T^{(1, \mathrm{PML})}\left(\mathbf{E}^{\mathrm{PML}}-\mathbf{E}_{\mathrm{I}}\right)_{\Gamma^{(1)}} \text { on } \Gamma^{(1)}, \\
\mathbf{H}^{\mathrm{PML}} \times \nu & =T^{(2, \mathrm{PML})}\left(\mathbf{E}^{\mathrm{PML}}\right)_{\Gamma^{(2)}} \text { on } \Gamma^{(2)} .
\end{aligned}
$$

Introduce the sesquilinear form $A^{\mathrm{PML}}: H_{\mathrm{qper}}(\operatorname{curl}, \Omega) \times H_{\mathrm{qper}}(\operatorname{curl}, \Omega) \rightarrow \mathbf{C}$,

$A^{\mathrm{PML}}(\varphi, \psi)=\int_{\Omega} \mu^{-1} \operatorname{curl} \varphi \cdot \operatorname{curl} \bar{\psi}-\int_{\Omega} \omega^{2} \varepsilon \varphi \cdot \bar{\psi}-\mathbf{i} \omega \sum_{j=1}^{2} \int_{\Gamma^{(j)}} T^{(j, \mathrm{PML})} \varphi_{\Gamma^{(j)}} \cdot \bar{\psi}_{\Gamma^{(j)}}$

and the following variational PML problem: Find $\mathbf{E}^{\mathrm{PML}} \in H_{\mathrm{qper}}(\operatorname{curl}, \Omega)$ such that

$$
A^{\mathrm{PML}}\left(\mathbf{E}^{\mathrm{PML}}, \psi\right)=\left\langle f_{\mathrm{I}}^{\mathrm{PML}}, \psi\right\rangle \quad \forall \psi \in H_{\mathrm{qper}}(\operatorname{curl}, \Omega),
$$

where

$$
\begin{aligned}
\left\langle f_{\mathrm{I}}^{\mathrm{PML}}, \psi\right\rangle & =\mathbf{i} \omega \int_{\Gamma^{(1)}}\left(\mathbf{H}_{\mathrm{I}} \times \nu-T^{(1, \mathrm{PML})}\left(\mathbf{E}_{\mathrm{I}}\right)_{\Gamma^{(1)}}\right) \cdot \bar{\psi}_{\Gamma^{(1)}} \\
& =-\mathbf{i} \omega \int_{\Gamma^{(1)}}\left(T^{(1)}\left(\mathbf{E}_{\mathrm{I}}\right)_{\Gamma^{(1)}}+T^{(1, \mathrm{PML})}\left(\mathbf{E}_{\mathrm{I}}\right)_{\Gamma^{(1)}}\right) \cdot \bar{\psi}_{\Gamma^{(1)}},
\end{aligned}
$$

where the identity (2.22) is used. The following lemma establishes the relationship between this variational problem and the weak formulation (2.34) straightforward from the above derivations, and hence the details of its proof are omitted.

Lemma 2.3. Any solution of the problem (2.34) restricted to $\Omega$ is a solution of (2.42). Conversely, any solution of the problem (2.42) can be uniquely extended to the whole domain $D$ as a solution of (2.34).

Next, we turn to estimate the error between $\mathbf{E}$ and $\mathbf{E}^{\mathrm{PML}}$. Clearly, it suffices to estimate the error between the capacity operators $T^{(j)}$ and $T^{(j, \mathrm{PML})}$. For $j=1,2$, let

$$
U^{(j)}=\left\{(l, m): \operatorname{Re}\left(\beta_{l, m}^{(j)}\right)^{2}=\omega^{2} \operatorname{Re}\left(\varepsilon^{(j)} \mu^{(j)}\right)-\left(\alpha_{1}^{l}\right)^{2}-\left(\alpha_{2}^{m}\right)^{2}>0\right\},
$$

and

$$
\begin{aligned}
& \beta_{+}^{(j)}=\min \left\{\left|\operatorname{Re}\left(\beta_{l, m}^{(j)}\right)^{2}\right|^{1 / 2}:(l, m) \in U^{(j)}\right\}, \\
& \beta_{-}^{(j)}=\min \left\{\left|\operatorname{Re}\left(\beta_{l, m}^{(j)}\right)^{2}\right|^{1 / 2}:(l, m) \notin U^{(j)}\right\} .
\end{aligned}
$$


Define $\beta_{+}^{(j)}=0$ if $U^{(j)}$ is empty. We have the following estimate for $\beta_{l, m}^{(j)}$.

Lemma 2.4. Let $s(\tau)=s_{1}(\tau)+\mathbf{i} s_{2}(\tau)$ be the model medium property satisfying (2.24). Then

$$
\begin{aligned}
& \operatorname{Re}\left(-\mathbf{i} \beta_{l, m}^{(j)} s(\tau)\right)=\operatorname{Re} \beta_{l, m}^{(j)} s_{2}(\tau)+\operatorname{Im} \beta_{l, m}^{(j)} s_{1}(\tau) \\
& \quad \geq \max \left\{\min \left(\beta_{+}^{(j)} s_{2}(\tau), \beta_{-}^{(j)} s_{1}(\tau)\right), s_{1}(\tau)\left(\frac{\omega^{2}}{2}\left(\left|\varepsilon^{(j)} \mu^{(j)}\right|-\operatorname{Re}\left(\varepsilon^{(j)} \mu^{(j)}\right)\right)\right)^{1 / 2}\right\} .
\end{aligned}
$$

Proof. From the definition (2.13) of $\beta_{l, m}^{(j)}$, we have $\operatorname{Im}\left(\left(\beta_{l, m}^{(j)}\right)^{2}\right)=\omega^{2} \operatorname{Im}\left(\varepsilon^{(j)} \mu^{(j)}\right)$, and hence

$$
\begin{aligned}
& \operatorname{Re} \beta_{l, m}^{(j)}=\frac{1}{\sqrt{2}}\left(\sqrt{\left(\omega^{2} \operatorname{Im}\left(\varepsilon^{(j)} \mu^{(j)}\right)\right)^{2}+\left(\operatorname{Re}\left(\beta_{l, m}^{(j)}\right)^{2}\right)^{2}}+\operatorname{Re}\left(\beta_{l, m}^{(j)}\right)^{2}\right)^{1 / 2} \\
& \operatorname{Im} \beta_{l, m}^{(j)}=\frac{1}{\sqrt{2}}\left(\sqrt{\left(\omega^{2} \operatorname{Im}\left(\varepsilon^{(j)} \mu^{(j)}\right)\right)^{2}+\left(\operatorname{Re}\left(\beta_{l, m}^{(j)}\right)^{2}\right)^{2}}-\operatorname{Re}\left(\beta_{l, m}^{(j)}\right)^{2}\right)^{1 / 2} .
\end{aligned}
$$

Since $\operatorname{Re}\left(\beta_{l, m}^{(j)}\right)^{2} \leq \omega^{2} \operatorname{Re}\left(\varepsilon^{(j)} \mu^{(j)}\right)$, this yields

$$
\operatorname{Im} \beta_{l, m}^{(j)} \geq\left(\frac{\omega^{2}}{2}\left(\left|\varepsilon^{(j)} \mu^{(j)}\right|-\operatorname{Re}\left(\varepsilon^{(j)} \mu^{(j)}\right)\right)\right)^{1 / 2} .
$$

Furthermore,

$$
\operatorname{Re} \beta_{l, m}^{(j)} \geq \beta_{+}^{(j)} \text { if } j \in U^{(j)} \quad \text { and } \quad \operatorname{Im} \beta_{l, m}^{(j)} \geq \beta_{-}^{(j)} \text { if } j \notin U^{(j)} .
$$

The proof is completed by combining the above two estimates.

The following lemma plays a key role in the subsequent analysis.

Lemma 2.5. For any $\varphi, \psi \in H_{\mathrm{qper}}(\operatorname{curl}, \Omega)$,

$$
\begin{aligned}
& \left|\omega \int_{\Gamma^{(j)}}\left(T^{(j)}-T^{(j, \mathrm{PML})}\right) \varphi_{\Gamma^{(j)}} \cdot \bar{\psi}_{\Gamma^{(j)}}\right| \\
& \leq M^{(j)}\left\|\varphi_{\Gamma^{(j)}}\right\|_{T H_{\mathrm{qper}}^{-1 / 2}\left(\operatorname{curl}, \Gamma^{(j)}\right)}\left\|\psi_{\Gamma^{(j)}}\right\|_{T H_{\mathrm{qper}}^{-1 / 2}\left(\operatorname{curl}, \Gamma^{(j)}\right)},
\end{aligned}
$$

where

$$
\begin{aligned}
& M^{(j)}=\frac{2 \max \left(\left|\omega^{2} \varepsilon^{(j)} \mu^{(j)}\right|, 1\right)}{\mu^{(j)}\left(e^{\Lambda^{(j)}}-1\right)} \\
& \quad \times \max \left\{\frac{\left(1+\omega^{2} \operatorname{Re}\left(\varepsilon^{(j)} \mu^{(j)}\right)-\left(\beta_{+}^{(j)}\right)^{2}\right)^{1 / 2}}{\left(\omega^{2} \operatorname{Im}\left(\varepsilon^{(j)} \mu^{(j)}\right)^{2}+\left(\beta_{+}^{(j)}\right)^{4}\right)^{1 / 4}}, \frac{\left(1+\omega^{2} \operatorname{Re}\left(\varepsilon^{(j)} \mu^{(j)}\right)+\left(\beta_{-}^{(j)}\right)^{2}\right)^{1 / 2}}{\left(\left(\omega^{2} \operatorname{Im}\left(\varepsilon^{(j)} \mu^{(j)}\right)\right)^{2}+\left(\beta_{-}^{(j)}\right)^{4}\right)^{1 / 4}}+1\right\}, \\
& \Lambda^{(j)}=\max \left\{2 \min \left(\beta_{+}^{(j)} \sigma_{I}^{(j)}, \beta_{-}^{(j)} \sigma_{R}^{(j)}\right), \sigma_{R}^{(j)}\left(2 \omega^{2}\left(\left|\varepsilon^{(j)} \mu^{(j)}\right|-\operatorname{Re}\left(\varepsilon^{(j)} \mu^{(j)}\right)\right)\right)^{1 / 2}\right\}, \\
& \text { and } \sigma_{R}^{(j)}, \sigma_{I}^{(j)} \text { are the real and imaginary parts of } \sigma^{(j)} \text { defined in }(\underline{2.39}), \text { i.e., } \sigma^{(j)}= \\
& \sigma_{R}^{(j)}+\mathbf{i} \sigma_{I}^{(j)} .
\end{aligned}
$$

Proof. For any $\varphi, \psi \in H_{\mathrm{qper}}(\operatorname{curl}, \Omega)$, their tangential components on $\Gamma^{(j)}$ have the following expansions:

$$
\begin{aligned}
& \varphi_{\Gamma^{(j)}}=\sum_{l, m \in Z}\left(\varphi_{l, m, 1}^{(j)}, \varphi_{l, m, 2}^{(j)}, 0\right)^{\mathrm{T}} e^{\mathbf{i}\left(\alpha_{1}^{l} x_{1}+\alpha_{2}^{m} x_{2}\right)}, \\
& \psi_{\Gamma^{(j)}}=\sum_{l, m \in Z}\left(\psi_{l, m, 1}^{(j)}, \psi_{l, m, 2}^{(j)}, 0\right)^{\mathrm{T}} e^{\mathbf{i}\left(\alpha_{1}^{l} x_{1}+\alpha_{2}^{m} x_{2}\right)} .
\end{aligned}
$$


It follows from the definitions of $T^{(j)}$ and $T^{(j, \mathrm{PML})}$ that

$$
\begin{aligned}
& \omega \int_{\Gamma^{(j)}}\left(T^{(j)}-T^{(j, \mathrm{PML})}\right) \varphi_{\Gamma^{(j)}} \cdot \bar{\psi}_{\Gamma^{(j)}} \mathrm{d} x_{1} \mathrm{~d} x_{2} \\
& =L_{1} L_{2} \sum_{l, m \in Z} \omega\left(r_{l, m, 1}^{(j)} \bar{\psi}_{l, m, 1}^{(j)}+r_{l, m, 2}^{(j)} \bar{\psi}_{l, m, 2}^{(j)}\right)\left(1-\operatorname{coth}\left(-\mathbf{i} \beta_{l, m}^{(j)} \sigma^{(j)}\right)\right) \\
& =L_{1} L_{2} \sum_{l, m \in Z} \frac{\left(1-\operatorname{coth}\left(-\mathbf{i} \beta_{l, m}^{(j)} \sigma^{(j)}\right)\right)}{\mu^{(j)} \beta_{l, m}^{(j)}}\left(\omega ^ { 2 } \varepsilon ^ { ( j ) } \mu ^ { ( j ) } \left(\varphi_{l, m, 1}^{(j)} \bar{\psi}_{l, m, 1}^{(j)}\right.\right. \\
& \left.\left.\quad+\varphi_{l, m, 2}^{(j)} \bar{\psi}_{l, m, 2}^{(j)}\right)-\left(\alpha_{1}^{l} \varphi_{l, m, 2}^{(j)}-\alpha_{2}^{m} \varphi_{l, m, 1}^{(j)}\right)\left(\alpha_{1}^{l} \bar{\psi}_{l, m, 2}^{(j)}-\alpha_{2}^{m} \bar{\psi}_{l, m, 1}^{(j)}\right)\right) .
\end{aligned}
$$

To prove the lemma, it is required to estimate

$$
\begin{aligned}
C_{l, m}^{(j)} & =\left|\left(1-\operatorname{coth}\left(-\mathbf{i} \beta_{l, m}^{(j)} \sigma^{(j)}\right)\right)\left(1+\left(\alpha_{1}^{l}\right)^{2}+\left(\alpha_{2}^{m}\right)^{2}\right)^{1 / 2} /\left(\mu^{(j)} \beta_{l, m}^{(j)}\right)\right| \\
& =\frac{2}{\mu^{(j)}\left|e^{-2 \mathbf{i} \beta_{l, m}^{(j)} \sigma^{(j)}}-1\right|}\left(\frac{\left(1+\omega^{2} \operatorname{Re}\left(\varepsilon^{(j)} \mu^{(j)}\right)-\operatorname{Re}\left(\beta_{l, m}^{(j)}\right)^{2}\right)^{2}}{\left(\omega^{2} \operatorname{Im}\left(\varepsilon^{(j)} \mu^{(j)}\right)\right)^{2}+\left(\operatorname{Re}\left(\beta_{l, m}^{(j)}\right)^{2}\right)^{2}}\right)^{1 / 4} .
\end{aligned}
$$

From Lemma 2.4

$$
\left|e^{-2 \mathbf{i} \beta_{l, m}^{(j)} \sigma^{(j)}}-1\right| \geq\left|e^{-2 \mathbf{i} \beta_{l, m}^{(j)} \sigma^{(j)}}\right|-1 \geq e^{\Lambda^{(j)}}-1 .
$$

Let $F^{(j)}(t)=\frac{\left(1+\omega^{2} \operatorname{Re}\left(\varepsilon^{(j)} \mu^{(j)}\right)-t\right)^{2}}{\left(\omega^{2} \operatorname{Im}\left(\varepsilon^{(j)} \mu^{(j)}\right)\right)^{2}+t^{2}}$. It can be verified that $F^{(j)}(t)$ increases for $t<K^{(j)}=-\left(\omega^{2} \operatorname{Im}\left(\varepsilon^{(j)} \mu^{(j)}\right)\right)^{2} /\left(\omega^{2} \operatorname{Re}\left(\varepsilon^{(j)} \mu^{j}\right)+1\right)$ and decreases for $K^{(j)}<$ $t \leq \omega^{2} \operatorname{Re}\left(\varepsilon^{(j)} \mu^{(j)}\right)$. Therefore,

$$
\begin{aligned}
& \frac{\left(1+\omega^{2} \operatorname{Re}\left(\varepsilon^{(j)} \mu^{(j)}\right)-\operatorname{Re}\left(\beta_{l, m}^{(j)}\right)^{2}\right)^{2}}{\left(\omega^{2} \operatorname{Im}\left(\varepsilon^{(j)} \mu^{(j)}\right)\right)^{2}+\left(\operatorname{Re}\left(\beta_{l, m}^{(j)}\right)^{2}\right)^{2}}=F^{(j)}\left(\operatorname{Re}\left(\beta_{l, m}^{(j)}\right)^{2}\right) \\
& \leq\left\{\begin{array}{c}
\max \left\{\frac{\left(1+\omega^{2} \operatorname{Re}\left(\varepsilon^{(j)} \mu^{(j)}\right)-\left(\beta_{+}^{(j)}\right)^{2}\right)^{2}}{\left(\omega^{2} \operatorname{Im}\left(\varepsilon^{(j)} \mu^{(j)}\right)\right)^{2}+\left(\beta_{+}^{(j)}\right)^{4}}, \frac{\left(1+\omega^{2} \operatorname{Re}\left(\varepsilon^{(j)} \mu^{(j)}\right)+\left(\beta_{-}^{(j)}\right)^{2}\right)^{2}}{\left(\omega^{2} \operatorname{Im}\left(\varepsilon^{(j)} \mu^{(j)}\right)\right)^{2}+\left(\beta_{-}^{(j)}\right)^{4}}\right\} \\
F\left(K^{(j)}\right)=\frac{\left(1+\omega^{2} \operatorname{Re}\left(\varepsilon^{(j)} \mu^{(j)}\right)\right)^{2}}{\left(\omega^{2} \operatorname{Im}\left(\varepsilon^{(j)}\right)(j)\right)^{2}}+1 \\
\leq \frac{\left(1+\omega^{2} \operatorname{Re}\left(\varepsilon^{(j)} \mu^{(j)}\right)\right)\left(1+\omega^{2} \operatorname{Re}\left(\varepsilon^{(j)} \mu^{(j)}\right)+\left(\beta_{-}^{(j)}\right)^{2}\right)}{\left(\omega^{2} \operatorname{Im}\left(\varepsilon^{(j)} \mu^{(j)}\right)\right)^{2}+\left(\beta_{-}^{(j)}\right)^{4}}+1 \\
\leq \max \left\{\frac{\left(1+\omega^{2} \operatorname{Re}\left(\varepsilon^{(j)} \mu^{(j)}\right)-\left(\beta_{+}^{(j)}\right)^{2}\right)^{2}}{\left(\omega^{2} \operatorname{Im}\left(\varepsilon^{(j)} \mu^{(j)}\right)\right)^{2}+\left(\beta_{+}^{(j)}\right)^{4}}, \frac{\left(1+\omega^{2} \operatorname{Re}\left(\varepsilon^{(j)} \mu^{(j)}\right)+\left(\beta_{-}^{(j)}\right)^{2}\right)^{2}}{\left(\omega^{2} \operatorname{Im}\left(\varepsilon^{(j)} \mu^{(j)}\right)\right)^{2}+\left(\beta_{-}^{(j)}\right)^{4}}+1\right.
\end{array}\right\} .
\end{aligned}
$$

We have by substituting (2.49) and (2.50) into (2.48) that

$$
\begin{aligned}
C_{l, m}^{(j)} \leq & \frac{2}{\mu^{(j)}\left(e^{\Lambda^{(j)}}-1\right)} \\
& \times \max \left\{\frac{\left(1+\omega^{2} \operatorname{Re}\left(\varepsilon^{(j)} \mu^{(j)}\right)-\left(\beta_{+}^{(j)}\right)^{2}\right)^{1 / 2}}{\left(\left(\omega^{2} \operatorname{Im}\left(\varepsilon^{(j)} \mu^{(j)}\right)\right)^{2}+\left(\beta_{+}^{(j)}\right)^{4}\right)^{1 / 4}}, \frac{\left(1+\omega^{2} \operatorname{Re}\left(\varepsilon^{(j)} \mu^{(j)}\right)+\left(\beta_{-}^{(j)}\right)^{2}\right)^{1 / 2}}{\left(\left(\omega^{2} \operatorname{Im}\left(\varepsilon^{(j)} \mu^{(j)}\right)\right)^{2}+\left(\beta_{-}^{(j)}\right)^{4}\right)^{1 / 4}}+1\right\} .
\end{aligned}
$$

The proof of the lemma follows from plugging this estimate into (2.47) and using the Cauchy-Schwarz inequality. 
Theorem 2.6. Let $\gamma_{0}$ and $\gamma_{1}$ be the constants in Lemma 2.2 and in the inf-sup condition (2.23), respectively. Suppose $\left(M^{(1)}+M^{(2)}\right)\left(\gamma_{0}\right)^{2}<\gamma_{1}$. Then the PML problem (2.34) has a unique solution $\mathbf{E}^{\mathrm{PML}}$. Moreover, it contains the following error estimate:

$$
\begin{aligned}
& \left\|\mathbf{E}-\mathbf{E}^{\mathrm{PML}}\right\| \|_{\Omega}:=\sup _{0 \neq \psi \in H_{\mathrm{qper}(\operatorname{curl}, \Omega)}} \frac{\left|A\left(\mathbf{E}-\mathbf{E}^{\mathrm{PML}}, \psi\right)\right|}{\|\psi\|_{H(\operatorname{curl}, \Omega)}} \\
& \leq \gamma_{0} M^{(1)}\left\|\mathbf{E}^{\mathrm{PML}}-\mathbf{E}_{\mathrm{I}}\right\|_{T H_{\mathrm{qper}}^{-1 / 2}\left(\operatorname{curl}, \Gamma^{(1)}\right)}+\gamma_{0} M^{(2)}\left\|\mathbf{E}^{\mathrm{PML}}\right\|_{T H_{\mathrm{qper}}^{-1 / 2}\left(\operatorname{curl}, \Gamma^{(2)}\right)} .
\end{aligned}
$$

Proof. By Lemma 2.3, it suffices to show that the variational problem (2.42) has a unique solution. We resort to the general existence and uniqueness results for sesquilinear forms in [5, Chapter 5]. The key point is to show the inf-sup condition for the sesquilinear form $A^{\mathrm{PML}}: H_{\mathrm{qper}}(\operatorname{curl}, \Omega) \times H_{\text {qper }}(\operatorname{curl}, \Omega) \rightarrow \mathbf{C}$ defined in (2.41). Due to Lemma 2.2 Lemma 2.5, and the assumption $\left(M^{(1)}+M^{(2)}\right)\left(\gamma_{0}\right)^{2}<$ $\gamma_{1}$, it is obvious: for any $\varphi, \psi \in H_{\mathrm{qper}}(\operatorname{curl}, \Omega)$,

$$
\begin{aligned}
\left|A^{\mathrm{PML}}(\varphi, \psi)\right| & \geq|A(\varphi, \psi)|-\sum_{j=1}^{2}\left|\omega \int_{\Gamma^{(j)}}\left(T^{(j)} \varphi_{\Gamma^{(j)}}-T^{(j, \mathrm{PML})} \varphi_{\Gamma^{(j)}}\right) \cdot \bar{\psi}_{\Gamma^{(j)}}\right| \\
& \geq|A(\varphi, \psi)|-\left(M^{(1)}+M^{(2)}\right)\left(\gamma_{0}\right)^{2}\|\varphi\|_{H(\operatorname{curl}, \Omega)}\|\psi\|_{H(\operatorname{curl}, \Omega)} .
\end{aligned}
$$

It remains to prove the estimate (2.51). By (2.19)-(2.21), (2.41)-(2.43), and Lemma 2.3 we conclude that

$$
\begin{aligned}
A\left(\mathbf{E}-\mathbf{E}^{\mathrm{PML}}, \psi\right)= & -\mathbf{i} \omega \int_{\Gamma^{(1)}}\left(T^{(1)}\left(\mathbf{E}_{\mathrm{I}}\right)_{\Gamma^{(1)}}-T^{(1, \mathrm{PML})}\left(\mathbf{E}_{\mathrm{I}}\right)_{\Gamma^{(1)}}\right) \cdot \bar{\psi}_{\Gamma^{(1)}} \\
& +A^{\mathrm{PML}}\left(\mathbf{E}^{\mathrm{PML}}, \psi\right)-A\left(\mathbf{E}^{\mathrm{PML}}, \psi\right) \\
= & \left.\mathbf{i} \omega \int_{\Gamma^{(1)}}\left(T^{(1)}-T^{(1, \mathrm{PML})}\right)\left(\mathbf{E}^{\mathrm{PML}}-\mathbf{E}_{\mathrm{I}}\right)_{\Gamma^{(1)}}\right) \cdot \bar{\psi}_{\Gamma^{(1)}} \\
& +\mathbf{i} \omega \int_{\Gamma^{(2)}}\left(T^{(2)}-T^{(2, \mathrm{PML})}\right) \mathbf{E}^{\mathrm{PML}} \cdot \bar{\psi}_{\Gamma^{(1)}}, \quad \forall \psi \in H_{\mathrm{qper}}(\operatorname{curl}, \Omega) .
\end{aligned}
$$

The proof is complete after using Lemma 2.5 and Lemma 2.2

Now let us take a closer look at the structure of the constant $M^{(j)}$, which controls the modeling error of the PML equation towards the original grating problem. Once the incoming plane wave is fixed, the numbers $\beta_{+}^{(j)}, \beta_{-}^{(j)}$ are fixed according to (2.45). Thus the constant $M^{(j)}$ approaches zero exponentially as the PML parameters $\sigma_{R}^{(j)}, \sigma_{I}^{(j)}$ tend to infinity. From the definition (2.39), the quantities $\sigma_{R}^{(j)}, \sigma_{I}^{(j)}$ can be calculated by the medium property $s(\tau)$, which is usually taken as a power function:

$$
s(\tau)=\left\{\begin{array}{ll}
1+\sigma_{m}^{(1)}\left(\frac{\tau-b^{(1)}}{\delta^{(1)}}\right)^{m} & \text { if } \tau \geq b^{(1)}, \\
1+\sigma_{m}^{(2)}\left(\frac{b^{(2)}-\tau}{\delta^{(2)}}\right)^{m} & \text { if } \tau \leq b^{(2)},
\end{array} \quad m \geq 1 .\right.
$$

Thus we have

$$
\sigma_{R}^{(j)}=\left(1+\frac{\operatorname{Re} \sigma_{m}^{(j)}}{m+1}\right) \delta^{(j)}, \quad \sigma_{I}^{(j)}=\frac{\operatorname{Im} \sigma_{m}^{(j)}}{m+1} \delta^{(j)} .
$$


It is obvious that either enlarging the thickness $\delta^{(j)}$ of the PML layers or enlarging the medium parameters $\operatorname{Re} \sigma_{m}^{(j)}$ and $\operatorname{Im} \sigma_{m}^{(j)}$ will reduce the PML approximation error.

\section{The DisCRETE PROBLEM}

In this section we introduce Nédeléc's element approximations 40] of the PML problems (2.34) and (2.42). Let $\mathcal{M}_{h}$ be a regular tetrahedral mesh of the domain $D$. Note that any tetrahedron $T \in \mathcal{M}_{h}$ is considered to be closed. We assume that any element $T$ must be completely included in $\overline{\left.\Omega^{(1, P M L}\right)}, \overline{\Omega^{(2, P M L)}}$, or $\bar{\Omega}$. To deal with the quasi-periodic boundary condition, we assume further that the mesh is periodic in both the $x_{1}$ and $x_{2}$ directions; that is, the projection of the surface mesh on any face of $D$ perpendicular to the $x_{1}$-axis (or the $x_{2}$-axis) into its opposite face coincides with the surface mesh on the opposite face. Denote by $\mathcal{F}_{h}$ the set of all faces of tetrahedrons in $\mathcal{M}_{h}$. Let $V_{h}(D) \subset \stackrel{\circ}{H_{\text {qper }}}(\operatorname{curl}, D)$ be an edge element space that contains the lowest order Nédélec's edge element space

$$
V_{h}^{1}(D)=\left\{v_{h} \in \stackrel{\circ}{H}_{\text {qper }}(\operatorname{curl}, D):\left.v_{h}\right|_{T}=a_{T}+b_{T} \times x, a_{T}, b_{T} \in \mathbb{C}^{3}, \forall T \in \mathcal{M}_{h}\right\} .
$$

To simplify the theoretical analysis, we first eliminate the nonhomogeneous Dirichlet boundary condition of the PML problem (2.34) by subtracting $\mathbf{E}_{\mathrm{I}}(x)-$ $\mathbf{E}_{\mathrm{I}}^{\mathbf{r}}(x)$ from $\mathbf{E}^{\mathrm{PML}}$ in $\Omega^{(1, \mathrm{PML})}$, where

$$
\begin{aligned}
& \operatorname{curl}\left(\hat{\mu}^{-1} \operatorname{curl} \mathbf{E}_{\mathrm{I}}^{\mathbf{r}}(x)\right)-\omega^{2} \hat{\varepsilon} \mathbf{E}_{\mathrm{I}}^{\mathbf{r}}(x)=0 \text { in } \Omega^{(1, \mathrm{PML})}, \\
& \mathbf{E}_{\mathrm{I}}^{\mathbf{r}} \times \nu=\mathbf{E}_{\mathrm{I}} \times \nu, \text { on } \Gamma^{(1)}, \quad \mathbf{E}_{\mathrm{I}}^{\mathbf{r}} \times \nu=0 \text { on } \Gamma^{(1, \mathrm{PML}) .} .
\end{aligned}
$$

We notice that $\mathbf{E}_{\mathrm{I}}^{\mathrm{r}}$ may be regarded as a mirror reflection of the incident field $\mathbf{E}_{\mathrm{I}}$ in the PML medium. Then the PML problem (2.34) can be rewritten as: Find

$$
\widetilde{\mathbf{E}}^{\mathrm{PML}}=\left\{\begin{array}{ll}
\mathbf{E}^{\mathrm{PML}}-\mathbf{E}_{\mathrm{I}}(x)+\mathbf{E}_{\mathrm{I}}^{\mathrm{r}}(x) & \text { in } \Omega^{(1, \mathrm{PML})}, \\
\mathbf{E}^{\mathrm{PML}} & \text { otherwise, }
\end{array} \widetilde{\mathbf{E}}^{\mathrm{PML}} \in \stackrel{\circ}{H_{\mathrm{qper}}}(\operatorname{curl}, D),\right.
$$

such that

$$
a_{D}\left(\widetilde{\mathbf{E}}^{\mathrm{PML}}, \psi\right)=\int_{\Gamma^{(1)}} \frac{1}{\mu^{(1)}} \operatorname{curl}\left(\mathbf{E}_{\mathrm{I}}-\mathbf{E}_{\mathrm{I}}^{\mathbf{r}}\right) \times \nu_{3} \cdot \bar{\psi}_{\Gamma^{(1)}}, \quad \forall \psi \in \stackrel{\circ}{H}_{\mathrm{qper}}(\operatorname{curl}, D),
$$

where $\nu_{3}=(0,0,1)^{\mathrm{T}}$. It follows from (3.2) and the definitions of $T^{(1)}(2.15)$ and $T^{(1, \mathrm{PML})}(2.38)$ that

$$
\begin{aligned}
& \frac{1}{\mu^{(1)}} \operatorname{curl}\left(\mathbf{E}_{\mathrm{I}}-\mathbf{E}_{\mathrm{I}}^{\mathbf{r}}\right) \times \nu_{3}=-\mathbf{i} \omega\left(T^{(1)}\left(\mathbf{E}_{\mathrm{I}}\right)_{\Gamma^{(1)}}+T^{(1, \mathrm{PML})}\left(\mathbf{E}_{\mathrm{I}}\right)_{\Gamma^{(1)}}\right) \\
& \quad=-\frac{\mathbf{i}}{\mu^{(1)}}\left(1+\operatorname{coth}\left(-\mathbf{i} \beta \sigma^{(1)}\right)\right)\left(p_{1} \beta+p_{3} \alpha_{1}, p_{2} \beta+p_{3} \alpha_{2}, 0\right)^{\mathrm{T}} e^{\mathbf{i} q \cdot x} \text { on } \Gamma^{(1)}
\end{aligned}
$$

where $\left(p_{1}, p_{2}, p_{3}\right)^{\mathrm{T}}=p$. We have from (2.43) that

$$
a_{D}\left(\widetilde{\mathbf{E}}^{\mathrm{PML}}, \psi\right)=\left\langle f_{\mathrm{I}}^{\mathrm{PML}}, \psi\right\rangle, \quad \forall \psi \in \stackrel{\circ}{H}_{\mathrm{qper}}(\operatorname{curl}, D) .
$$

The edge element approximation to the PML problem (3.4) reads as follows: Find $\widetilde{\mathbf{E}}_{h}^{\mathrm{PML}} \in V_{h}(D)$ such that

$$
a_{D}\left(\widetilde{\mathbf{E}}_{h}^{\mathrm{PML}}, \psi_{h}\right)=\left\langle f_{\mathrm{I}}^{\mathrm{PML}}, \psi_{h}\right\rangle, \quad \forall \psi_{h} \in V_{h}(D) .
$$

The discrete problem (3.7) is assumed to attain a unique solution $\widetilde{\mathbf{E}}_{h}^{\mathrm{PML}} \in V_{h}(D)$. 
Given a face $F \in \mathcal{F}_{h}, h_{F}$ stands for its length. For any $T \in \mathcal{M}_{h}$, we denote by $h_{T}$ its diameter and introduce the residuals

$$
\begin{aligned}
R_{T}^{(1)} & :=\left.\omega^{2} \hat{\varepsilon} \widetilde{\mathbf{E}}_{h}^{\mathrm{PML}}\right|_{T}-\operatorname{curl}\left(\left.\hat{\mu}^{-1} \operatorname{curl} \widetilde{\mathbf{E}}_{h}^{\mathrm{PML}}\right|_{T}\right), \\
R_{T}^{(2)} & :=-\operatorname{div}\left(\left.\omega^{2} \hat{\varepsilon} \widetilde{\mathbf{E}}_{h}^{\mathrm{PML}}\right|_{T}\right) .
\end{aligned}
$$

Given an interior side $F \in \mathcal{F}_{h}$, which is the common side of $T_{1}$ and $T_{2} \in \mathcal{M}_{h}$, we define the jump residuals across $F$ as

$$
\begin{aligned}
& J_{F}^{(1)}= \begin{cases}\hat{\mu}^{-1}\left(\left.\operatorname{curl} \widetilde{\mathbf{E}}_{h}^{\mathrm{PML}}\right|_{T_{1}}-\left.\operatorname{curl} \widetilde{\mathbf{E}}_{h}^{\mathrm{PML}}\right|_{T_{2}}\right) \times \nu_{F}, & \text { if } F \not \subset \Gamma^{(1)}, \\
\frac{\left(\left.\operatorname{curl} \widetilde{\mathbf{E}}_{h}^{\mathrm{PML}}\right|_{T_{1}}-\left.\operatorname{curl} \widetilde{\mathbf{E}}_{h}^{\mathrm{PML}}\right|_{T_{2}}\right) \times \nu_{F}}{\mu^{(1)}}+\frac{\operatorname{curl}\left(\mathbf{E}_{\mathrm{I}}-\mathbf{E}_{\mathrm{I}}^{\mathrm{r}}\right) \times \nu_{3}}{\mu^{(1)}}, & \text { if } F \subset \Gamma^{(1)},\end{cases} \\
& J_{F}^{(2)}= \begin{cases}\omega^{2}\left(\left.\hat{\varepsilon}_{h}^{\mathrm{E}} \widetilde{\mathbf{E}}_{h}^{\mathrm{PL}}\right|_{T_{2}}-\left.\hat{\varepsilon} \widetilde{\mathbf{E}}_{h}^{\mathrm{PML}}\right|_{T_{1}}\right) \cdot \nu_{F}, & \text { if } F \not \subset \Gamma^{(1)}, \\
\omega^{2} \varepsilon\left(\left.\widetilde{\mathbf{E}}_{h}^{\mathrm{PML}}\right|_{T_{2}}-\left.\widetilde{\mathbf{E}}_{h}^{\mathrm{PML}}\right|_{T_{1}}\right) \cdot \nu_{F}-\omega^{2} \varepsilon\left(\mathbf{E}_{\mathrm{I}}-\mathbf{E}_{\mathrm{I}}^{\mathbf{r}}\right) \cdot \nu_{3}, & \text { if } F \subset \Gamma^{(1)} .\end{cases}
\end{aligned}
$$

Here $\nu_{3}=(0,0,1)^{\mathrm{T}}$ and the unit normal vector $\nu_{F}$ to $F$ points from $T_{2}$ to $T_{1}$. Define

$$
\begin{aligned}
& \Gamma_{10}=\left\{\left(x_{1}, x_{2}, x_{3}\right): x_{1}=0,0<x_{2}<L_{2}, b^{(2)}-\delta^{(2)}<x_{3}<b^{(1)}+\delta^{(1)}\right\}, \\
& \Gamma_{11}=\left\{\left(x_{1}, x_{2}, x_{3}\right): x_{1}=L_{1}, 0<x_{2}<L_{2}, b^{(2)}-\delta^{(2)}<x_{3}<b^{(1)}+\delta^{(1)}\right\}, \\
& \Gamma_{20}=\left\{\left(x_{1}, x_{2}, x_{3}\right): x_{2}=0,0<x_{1}<L_{1}, b^{(2)}-\delta^{(2)}<x_{3}<b^{(1)}+\delta^{(1)}\right\}, \\
& \Gamma_{21}=\left\{\left(x_{1}, x_{2}, x_{3}\right): x_{2}=L_{2}, 0<x_{1}<L_{1}, b^{(2)}-\delta^{(2)}<x_{3}<b^{(1)}+\delta^{(1)}\right\} .
\end{aligned}
$$

For some element $T \in \mathcal{M}_{h}$, if $F=\Gamma_{j 0} \cap \partial T$ and the corresponding face $F^{\prime}$ on $\Gamma_{j 1}$ is a face of some element $T^{\prime}$, then we define the jump residual as

$$
\begin{aligned}
J_{F}^{(1)} & =\hat{\mu}^{-1}\left(\left.\operatorname{curl} \widetilde{\mathbf{E}}_{h}^{\mathrm{PML}}\right|_{T}-\left.e^{-\mathbf{i} \alpha_{j} L_{j}} \operatorname{curl} \widetilde{\mathbf{E}}_{h}^{\mathrm{PML}}\right|_{T^{\prime}}\right) \times \nu_{j}, \\
J_{F^{\prime}}^{(1)} & =\hat{\mu}^{-1}\left(\left.e^{\mathbf{i} \alpha_{j} L_{j}} \operatorname{curl} \widetilde{\mathbf{E}}_{h}^{\mathrm{PML}}\right|_{T}-\left.\operatorname{curl} \widetilde{\mathbf{E}}_{h}^{\mathrm{PML}}\right|_{T^{\prime}}\right) \times \nu_{j} .
\end{aligned}
$$

Here $\nu_{1}=(1,0,0)^{\mathrm{T}}$ and $\nu_{2}=(0,1,0)^{\mathrm{T}}$. Moreover, we define

$$
J_{F}^{(1)}=(0,0,0)^{\mathrm{T}} \text { if } F \subset \overline{\Gamma^{(1, \mathrm{PML})} \cup \Gamma^{(2, \mathrm{PML})}} \quad \text { and } \quad J_{F}^{(2)}=0 \text { if } F \subset \partial D .
$$

For any $T \in \mathcal{M}_{h}$, denote by $\eta_{T}$ the local error estimator, which is defined as follows:

$$
\begin{aligned}
\eta_{T}= & h_{T}\left(\left\|R_{T}^{(1)}\right\|_{\left(L^{2}(T)\right)^{3}}^{2}+\left\|R_{T}^{(2)}\right\|_{L^{2}(T)}^{2}\right)^{1 / 2} \\
& +\left(\frac{1}{2} \sum_{F \subset \partial T} h_{F}\left(\left\|J_{F}^{(1)}\right\|_{\left(L^{2}(F)\right)^{3}}^{2}+\left\|J_{F}^{(2)}\right\|_{L^{2}(F)}^{2}\right)\right)^{1 / 2} .
\end{aligned}
$$

The following theorem is the main result in this paper.

Theorem 3.1. There exists a constant $C>0$, depending only on the minimum angle of the mesh $\mathcal{M}_{h}$, such that the following a posteriori error estimate holds:

$$
\begin{aligned}
\left\|\mathbf{E}-\widetilde{\mathbf{E}}_{h}^{\mathrm{PML}}\right\| \|_{\Omega} \leq & \gamma_{0}^{2} M^{(1)}\left\|\widetilde{\mathbf{E}}_{h}^{\mathrm{PML}}-\mathbf{E}_{\mathrm{I}}\right\|_{H(\operatorname{curl}, \Omega)}+\gamma_{0}^{2} M^{(2)}\left\|\widetilde{\mathbf{E}}_{h}^{\mathrm{PML}}\right\|_{H(\operatorname{curl}, \Omega)} \\
& +C\left[1+\gamma_{0} \max \left|s\left(x_{3}\right)\right|\left(C^{(1)}+C^{(2)}\right)\right]\left(\sum_{T \in \mathcal{M}_{h}} \eta_{T}^{2}\right)^{1 / 2},
\end{aligned}
$$

where the constants $\gamma_{0}, M^{(j)}$, and $C^{(j)}, j=1,2$ are defined in Lemmas 2.2, 2.5, and 4.3 , respectively. 
The proof of this theorem will be given in Section 4. We notice that when the PML parameters $\sigma_{R}^{(j)}$ and $\sigma_{I}^{(j)}$ tend to infinity, the constants $M^{(j)}$ decay exponentially and the constants $C^{(j)}$ remain bounded.

\section{A POSTERIORI ERROR ANALYSIS}

In this section we prove the a posteriori error estimate of Theorem 3.1 .

4.1. Error representation formula. For any $\psi \in H_{\mathrm{qper}}(\operatorname{curl}, \Omega)$, we extend it to be a function in $\stackrel{\circ}{H}_{\text {qper }}(\operatorname{curl}, D)$. Denote the extension by $\tilde{\psi}$ such that $\tilde{\psi}=\psi$ in $\Omega$ and

$$
\begin{aligned}
\operatorname{curl}\left(\hat{\mu}^{-1} \operatorname{curl} \overline{\tilde{\psi}}\right)-\omega^{2} \hat{\varepsilon} \bar{\psi} & =0 \quad \text { in } \Omega^{(j, \mathrm{PML})}, \\
\tilde{\psi} \times \nu^{(j)} & =\psi \times \nu^{(j)} \quad \text { on } \Gamma^{(j)}, \quad j=1,2,
\end{aligned}
$$

where $\nu^{(j)}$ is the unit outer normal to $\Omega^{(j, \mathrm{PML})}$.

Lemma 4.1. For any $\varphi$ and $\psi \in H_{\mathrm{qper}}(\operatorname{curl}, \Omega)$,

$$
\mathbf{i} \omega \int_{\Gamma^{(j)}} T^{(j, \mathrm{PML})} \varphi_{\Gamma^{(j)}} \cdot \bar{\psi}_{\Gamma^{(j)}}=\int_{\Gamma^{(j)}} \frac{1}{\mu^{(j)}}\left(\varphi \times \nu^{(j)}\right) \cdot(\operatorname{curl} \overline{\tilde{\psi}})_{\Gamma^{(j)}} .
$$

Proof. Define $\phi \in H_{\mathrm{qper}}\left(\mathrm{curl}, \Omega^{(j, \mathrm{PML})}\right)$ as follows:

$$
\left\{\begin{array}{l}
\operatorname{curl}\left(\hat{\mu}^{-1} \operatorname{curl} \phi\right)-\omega^{2} \hat{\varepsilon} \phi=0 \quad \text { in } \Omega^{(j, \mathrm{PML})} \\
\phi \times \nu^{(j)}=\varphi \times \nu^{(j)} \text { on } \Gamma^{(j)}, \quad \text { and } \phi \times \nu^{(j)}=0 \text { on } \Gamma^{(j, \mathrm{PML})} .
\end{array}\right.
$$

It is easy to verify that (see (2.29) and (2.40) $)$

$$
\frac{1}{\mu^{(j)}} \operatorname{curl} \phi \times \nu^{(j)}=-\mathbf{i} \omega T^{(j, \mathrm{PML})} \varphi_{\Gamma^{(j)}} \text { on } \Gamma^{(j)} .
$$

By noting that $\hat{\mu}^{-1}$ is diagonal in $\Omega^{(j, \mathrm{PML})}$ and $\hat{\mu}^{-1}=\left(\mu^{(j)}\right)^{-1} I$ on $\Gamma^{(j)}$ we conclude by integration by parts that

$$
\begin{aligned}
\int_{\Gamma^{(j)}} \frac{1}{\mu^{(j)}} & \left(\phi \times \nu^{(j)}\right) \cdot(\operatorname{curl} \overline{\tilde{\psi}})_{\Gamma^{(j)}}=-\int_{\Gamma^{(j)}} \frac{1}{\mu^{(j)}}\left(\operatorname{curl} \overline{\tilde{\psi}} \times \nu^{(j)}\right) \cdot\left(\phi_{\Gamma^{(j)}}\right) \\
& =-\int_{\Omega^{(j, \mathrm{PML})}}\left(\hat{\mu}^{-1} \operatorname{curl} \overline{\tilde{\psi}} \cdot \operatorname{curl} \phi-\omega^{2} \hat{\varepsilon} \tilde{\tilde{\psi}} \cdot \phi\right) \\
& =-\int_{\Omega^{(j, \mathrm{PML})}}\left(\hat{\mu}^{-1} \operatorname{curl} \phi \cdot \operatorname{curl} \overline{\tilde{\psi}}-\omega^{2} \hat{\varepsilon} \phi \cdot \overline{\tilde{\psi}}\right) \\
& =-\int_{\Gamma^{(j)}} \frac{1}{\mu^{(j)}}\left(\operatorname{curl} \phi \times \nu^{(j)}\right) \cdot\left(\overline{\tilde{\psi}}_{\Gamma^{(j)}}\right)=\mathbf{i} \omega \int_{\Gamma^{(j)}} T^{(j, \mathrm{PML})} \varphi_{\Gamma^{(j)}} \cdot \bar{\psi}_{\Gamma^{(j)}},
\end{aligned}
$$

which completes the proof.

From now on, for convenience, we denote $\tilde{\psi}$, the PML extension of $\psi$, also by $\psi$.

Lemma 4.2 (The error representational formula). For any $\psi \in H_{\mathrm{qper}}(\operatorname{curl}, \Omega)$, which is extended to be a function in $\stackrel{\circ}{H}_{\mathrm{qper}}(\mathrm{curl}, D)$ according to (4.1), and $\psi_{h} \in$ $V_{h}(D)$, 


$$
\begin{aligned}
A\left(\mathbf{E}-\widetilde{\mathbf{E}}_{h}^{\mathrm{PML}}, \psi\right)= & \int_{\Gamma^{(1)}} \frac{1}{\mu^{(1)}} \operatorname{curl}\left(\mathbf{E}_{\mathrm{I}}-\mathbf{E}_{\mathrm{I}}^{\mathbf{r}}\right) \times \nu_{3} \cdot\left(\overline{\psi-\psi_{h}}\right)_{\Gamma^{(1)}}-a_{D}\left(\widetilde{\mathbf{E}}_{h}^{\mathrm{PML}}, \psi-\psi_{h}\right) \\
& +\mathbf{i} \omega \int_{\Gamma^{(1)}}\left(T^{(1)}-T^{(1, \mathrm{PML})}\right)\left(\widetilde{\mathbf{E}}_{h}^{\mathrm{PML}}-\mathbf{E}_{\mathrm{I}}\right)_{\Gamma^{(1)}} \cdot \bar{\psi}_{\Gamma^{(1)}} \\
& +\mathbf{i} \omega \int_{\Gamma^{(2)}}\left(T^{(2)}-T^{(2, \mathrm{PML})}\right)\left(\widetilde{\mathbf{E}}_{h}^{\mathrm{PML}}\right)_{\Gamma^{(2)}} \cdot \bar{\psi}_{\Gamma^{(2)}} .
\end{aligned}
$$

Proof. First from (2.19)-(2.21), (2.41)-(2.43), and (3.6) we have

$$
\begin{aligned}
A\left(\mathbf{E}-\widetilde{\mathbf{E}}_{h}^{\mathrm{PML}}, \psi\right)= & A(\mathbf{E}, \psi)-A^{\mathrm{PML}}\left(\mathbf{E}^{\mathrm{PML}}, \psi\right)+A^{\mathrm{PML}}\left(\widetilde{\mathbf{E}}_{h}^{\mathrm{PML}}, \psi\right)-A\left(\widetilde{\mathbf{E}}_{h}^{\mathrm{PML}}, \psi\right) \\
& +A^{\mathrm{PML}}\left(\mathbf{E}^{\mathrm{PML}}, \psi\right)-A^{\mathrm{PML}}\left(\widetilde{\mathbf{E}}_{h}^{\mathrm{PML}}, \psi\right) \\
= & \left\langle f_{\mathrm{I}}-f_{\mathrm{I}}^{\mathrm{PML}}, \psi\right\rangle+\mathbf{i} \omega \sum_{j=1}^{2} \int_{\Gamma^{(j)}}\left(T^{(j)}-T^{(j, \mathrm{PML})}\right)\left(\widetilde{\mathbf{E}}_{h}^{\mathrm{PML}}\right)_{\Gamma^{(j)}} \cdot \bar{\psi}_{\Gamma^{(j)}} \\
& +a_{D}\left(\widetilde{\mathbf{E}}^{\mathrm{PML}}, \psi\right)-A^{\mathrm{PML}}\left(\widetilde{\mathbf{E}}_{h}^{\mathrm{PML}}, \psi\right) \\
= & \mathbf{i} \omega \int_{\Gamma^{(1)}}\left(T^{(1)}-T^{(1, \mathrm{PML})}\right)\left(\widetilde{\mathbf{E}}_{h}^{\mathrm{PML}}-\mathbf{E}_{\mathrm{I}}\right)_{\Gamma^{(1)}} \cdot \bar{\psi}_{\Gamma^{(1)}} \\
& +\mathbf{i} \omega \int_{\Gamma^{(2)}}\left(T^{(2)}-T^{(2, \mathrm{PML})}\right)\left(\widetilde{\mathbf{E}}_{h}^{\mathrm{PML}}\right)_{\Gamma^{(2)}} \cdot \bar{\psi}_{\Gamma^{(2)}} \\
& +a_{D}\left(\widetilde{\mathbf{E}}^{\mathrm{PML}}, \psi\right)-A^{\mathrm{PML}}\left(\widetilde{\mathbf{E}}_{h}^{\mathrm{PML}}, \psi\right) .
\end{aligned}
$$

It follows from (2.33), Lemma 4.1, (4.1), and integration by parts that

$$
\begin{aligned}
A^{\mathrm{PML}}\left(\widetilde{\mathbf{E}}_{h}^{\mathrm{PML}}, \psi\right) & =a_{\Omega}\left(\widetilde{\mathbf{E}}_{h}^{\mathrm{PML}}, \psi\right)-\mathbf{i} \omega \sum_{j=1}^{2} \int_{\Gamma^{(j)}} T^{(j, \mathrm{PML})}\left(\widetilde{\mathbf{E}}_{h}^{\mathrm{PML}}\right)_{\Gamma^{(j)}} \cdot \bar{\psi}_{\Gamma^{(j)}} \\
& =a_{\Omega}\left(\widetilde{\mathbf{E}}_{h}^{\mathrm{PML}}, \psi\right)-\sum_{j=1}^{2} \int_{\Gamma^{(j)}} \frac{1}{\mu^{(j)}}\left(\widetilde{\mathbf{E}}_{h}^{\mathrm{PML}}\right) \times \nu^{(j)} \cdot(\operatorname{curl} \bar{\psi})_{\Gamma^{(j)}} \\
& =a_{\Omega}\left(\widetilde{\mathbf{E}}_{h}^{\mathrm{PML}}, \psi\right)+\sum_{j=1}^{2} a_{\Omega^{(j, \mathrm{PML})}}\left(\widetilde{\mathbf{E}}_{h}^{\mathrm{PML}}, \psi\right) \\
& =a_{D}\left(\widetilde{\mathbf{E}}_{h}^{\mathrm{PML}}, \psi\right),
\end{aligned}
$$

which completes the proof by using (3.6) and (3.7).

We remark that evaluating the various terms in the error representation formula would yield the desired a posteriori error estimate in Theorem 3.1. To achieve this goal, we need to prove stability estimates for the extension (4.1) of the function $\psi$ in $\Omega^{(j, \mathrm{PML})}$. We have the following lemma, whose proof will be given in Appendix A.1.

Lemma 4.3. For any $\psi \in H_{\mathrm{qper}}(\operatorname{curl}, \Omega)$, let $\psi$ be extended to the whole domain $D$ according to (4.1). Then the following estimates hold, for $j=1,2$ :

$$
\left(\left\|s^{-1} \psi\right\|_{\left(L^{2}\left(\Omega^{(j, \mathrm{PML})}\right)\right)^{3}}^{2}+\left\|s^{-1} \operatorname{curl} \psi\right\|_{\left(L^{2}\left(\Omega^{(j, \mathrm{PML})}\right)\right)^{3}}^{2}\right)^{1 / 2} \leq \gamma_{0} C^{(j)}\|\psi\|_{H(\operatorname{curl}, \Omega)},
$$


where $\gamma_{0}$ is defined in Lemma 2.2 and

$$
C^{(j)}=\frac{\sqrt[4]{16\left(\delta^{(j)}\right)^{2}\left(1+\omega^{2} \operatorname{Re}\left(\varepsilon^{(j)} \mu^{(j)}\right)\right)+4}}{1-e^{-\Lambda^{(j)}}} \sqrt{\frac{\omega^{2} \operatorname{Re}\left(\varepsilon^{(j)} \mu^{(j)}\right)+\left|\omega^{2} \varepsilon^{(j)} \mu^{(j)}\right|^{2}}{\sqrt{\left(\omega^{2} \operatorname{Im}\left(\varepsilon^{(j)} \mu^{(j)}\right)\right)^{2}+\min \left\{\left(\beta_{+}^{(j)}\right)^{4},\left(\beta_{-}^{(j)}\right)^{4}\right\}}}+2},
$$

where $\Lambda^{(j)}$ is defined in Lemma 2.5.

4.2. Proof of Theorem 3.1. Noticing that $D$ is a cuboid, we have the following Hodge decomposition (cf. Theorem 3.45 in [36) : For any $\psi \in \stackrel{\circ}{H}_{\text {qper }}(\operatorname{curl}, D)$, there exist $\psi^{(1)} \in\left(H^{1}(D)\right)^{3}$ and $\psi^{(2)} \in H_{0}^{1}(D)$ such that

$$
\psi=\psi^{(1)}+\operatorname{grad} \psi^{(2)}, \quad \operatorname{div} \psi^{(1)}=0,
$$

and by a similar argument as Theorem 2.3 in [26] the following identity holds:

$$
\left\|\psi^{(1)}\right\|_{\left(H^{1}(D)\right)^{3}}=\left\|\psi^{(1)}\right\|_{H(\operatorname{curl}, D)} .
$$

It is clear that

$$
\begin{aligned}
\left|\psi^{(1)}\right|_{\left(H^{1}(D)\right)^{3}}^{2} & +\left|\psi^{(2)}\right|_{H^{1}(D)}^{2} \leq\left\|\psi^{(1)}\right\|_{H(\operatorname{curl}, D)}^{2}+\left|\psi^{(2)}\right|_{H^{1}(D)}^{2} \\
& =\left\|\operatorname{curl} \psi^{(1)}\right\|_{\left(L^{2}(D)\right)^{3}}^{2}+\left\|\psi^{(1)}\right\|_{\left(L^{2}(D)\right)^{3}}^{2}+\left|\psi^{(2)}\right|_{H^{1}(D)}^{2} \\
& =\|\operatorname{curl} \psi\|_{\left(L^{2}(D)\right)^{3}}^{2}+\|\psi\|_{\left(L^{2}(D)\right)^{3}}^{2}=\|\psi\|_{H(\operatorname{curl}, D)}^{2}
\end{aligned}
$$

Notice that $\operatorname{grad} \psi^{(2)} \times \nu=0$ on $\partial D$, where $\nu$ is the unit outer normal to $\partial D$. Since $\psi \in \stackrel{\circ}{H}_{\text {qper }}(\operatorname{curl}, D)$, we have from (2.4) that $\psi^{(1)} \in \stackrel{\circ}{H}_{\text {qper }}(\operatorname{curl}, D) \cap\left(H^{1}(D)\right)^{3}$.

Denote by $U_{h}(D) \subset H_{0}^{1}(D)$ the standard continuous piecewise linear finite element space. Clearly,

$$
\operatorname{grad} U_{h}(D) \subseteq V_{h}^{1}(D)
$$

Here $V_{h}^{1}(D)$ is the lowest order Nédélec's edge element space defined in (3.1). For any element $T \in \mathcal{M}_{h}$, denote by $\widetilde{T}$ the point sets that contain all elements in $\mathcal{M}_{h}$ sharing at least one vertex with $T$. For any face $F \in \mathcal{F}_{h}$, denote by $\widetilde{F}=\bigcup\{\widetilde{T}$ : $F \subset \partial T\}$. Let $P_{h}: H_{0}^{1}(D) \mapsto U_{h}(D)$ be the Scott-Zhang interpolation operator 43. Then

$$
\begin{aligned}
& \left\|\psi^{(2)}-P_{h} \psi^{(2)}\right\|_{L^{2}(T)} \leq C h_{T}\left|\psi^{(2)}\right|_{H^{1}(\widetilde{T})}, \\
& \left\|\psi^{(2)}-P_{h} \psi^{(2)}\right\|_{L^{2}(F)} \leq C h_{F}^{1 / 2}\left|\psi^{(2)}\right|_{H^{1}(\widetilde{F})} .
\end{aligned}
$$

Here $C$ is a constant depending only on the minimum angle of the mesh, and $h_{T}$ and $h_{F}$ are the diameters of $T$ and $F$, respectively.

The following lemma introduces an interpolation operator $Q_{h}: \stackrel{\circ}{H_{\text {qper }}}(\operatorname{curl}, D) \cap$ $\left(H^{1}(D)\right)^{3} \mapsto V_{h}^{1}(D)$ satisfying the estimates (4.8) and (4.9). The operator is similar to the operator introduced by Beck, Hiptmair, Hoppe, and Wohlmuth 14 for the non-quasi-periodic boundary condition. The proof of the lemma is given in Appendix A.2. 
Lemma 4.4. There exists a linear projection $Q_{h}: \stackrel{\circ}{H}_{\mathrm{qper}}(\operatorname{curl}, D) \cap\left(H^{1}(D)\right)^{3} \mapsto$ $V_{h}^{1}(D)$ satisfying the following estimates:

$$
\begin{aligned}
& \left\|Q_{h} \psi^{(1)}\right\|_{\left(L^{2}(T)\right)^{3}} \leq C\left(\left\|\psi^{(1)}\right\|_{\left(L^{2}(\widetilde{T})\right)^{3}}+h_{T}\left|\psi^{(1)}\right|_{\left(H^{1}(\widetilde{T})\right)^{3}}\right), \\
& \left\|\psi^{(1)}-Q_{h} \psi^{(1)}\right\|_{\left(L^{2}(T)\right)^{3}} \leq C h_{T}\left|\psi^{(1)}\right|_{\left(H^{1}(\widetilde{T})\right)^{3}}, \\
& \left\|\psi^{(1)}-Q_{h} \psi^{(1)}\right\|_{\left(L^{2}(F)\right)^{3}} \leq C h_{F}^{1 / 2}\left|\psi^{(1)}\right|_{\left(H^{1}(\widetilde{F})\right)^{3}} .
\end{aligned}
$$

Next we turn to the a posteriori error estimate. For any $\psi=\psi^{(1)}+\operatorname{grad} \psi^{(2)}$ satisfying the Hodge decomposition (4.3), let

$$
\psi_{h}^{(1)}=Q_{h} \psi^{(1)}, \quad \psi_{h}^{(2)}=P_{h} \psi^{(2)}, \quad \psi_{h}=\psi_{h}^{(1)}+\operatorname{grad} \psi_{h}^{(2)} .
$$

It follows from the error representation formula (Lemma 4.2) that

$$
\begin{aligned}
A\left(\mathbf{E}-\widetilde{\mathbf{E}}_{h}^{\mathrm{PML}}, \psi\right) & \left.=\int_{\Gamma^{(1)}} \frac{1}{\mu^{(1)}} \operatorname{curl}\left(\mathbf{E}_{\mathrm{I}}-\mathbf{E}_{\mathrm{I}}^{\mathrm{r}}\right) \times \nu_{3} \cdot \overline{\left(\psi^{(1)}-\psi_{h}^{(1)}+\operatorname{grad}\left(\psi^{(2)}-\psi_{h}^{(2)}\right)\right.}\right)_{\Gamma^{(1)}} \\
& -a_{D}\left(\widetilde{\mathbf{E}}_{h}^{\mathrm{PML}}, \psi^{(1)}-\psi_{h}^{(1)}+\operatorname{grad}\left(\psi^{(2)}-\psi_{h}^{(2)}\right)\right) \\
& +\mathbf{i} \omega \int_{\Gamma^{(1)}}\left(T^{(1)}-T^{(1, \mathrm{PML})}\right)\left(\widetilde{\mathbf{E}}_{h}^{\mathrm{PML}}-\mathbf{E}_{\mathrm{I}}\right)_{\Gamma^{(1)}} \cdot \bar{\psi}_{\Gamma^{(1)}} \\
& +\mathbf{i} \omega \int_{\Gamma^{(2)}}\left(T^{(2)}-T^{(2, \mathrm{PML})}\right)\left(\widetilde{\mathbf{E}}_{h}^{\mathrm{PML}}\right)_{\Gamma^{(2)}} \cdot \bar{\psi}_{\Gamma^{(2)}} \\
:= & \mathrm{II}+\mathrm{III}+\mathrm{IV}+\mathrm{V} .
\end{aligned}
$$

First, we have from (2.33) that

$$
\begin{aligned}
\mathrm{II}+\mathrm{III}= & \left.\int_{\Gamma^{(1)}} \frac{1}{\mu^{(1)}} \operatorname{curl}\left(\mathbf{E}_{\mathrm{I}}-\mathbf{E}_{\mathrm{I}}^{\mathbf{r}}\right) \times \nu_{3} \cdot \overline{\left(\psi^{(1)}-\psi_{h}^{(1)}+\operatorname{grad}\left(\psi^{(2)}-\psi_{h}^{(2)}\right)\right.}\right)_{\Gamma^{(1)}} \\
& +\int_{D} \omega^{2} \hat{\varepsilon} \widetilde{\mathbf{E}}_{h}^{\mathrm{PML}} \cdot\left(\overline{\psi^{(1)}-\psi_{h}^{(1)}}+\operatorname{grad} \overline{\left(\psi^{(2)}-\psi_{h}^{(2)}\right)}\right) \\
& -\int_{D} \hat{\mu}^{-1} \operatorname{curl} \widetilde{\mathbf{E}}_{h}^{\mathrm{PML}} \cdot \operatorname{curl} \overline{\left(\psi^{(1)}-\psi_{h}^{(1)}\right)} \\
= & \int_{\Gamma^{(1)}} \frac{1}{\mu^{(1)}} \operatorname{curl}\left(\mathbf{E}_{\mathrm{I}}-\mathbf{E}_{\mathrm{I}}^{\mathbf{r}}\right) \times \nu_{3} \cdot \overline{\left(\overline{\psi^{(1)}-\psi_{h}^{(1)}}\right)_{\Gamma^{(1)}}} \\
& \left.+\int_{\Gamma^{(1)}} \frac{1}{\mu^{(1)}} \operatorname{curl}\left(\mathbf{E}_{\mathrm{I}}-\mathbf{E}_{\mathrm{I}}^{\mathbf{r}}\right) \times \nu_{3} \cdot \overline{\left(\operatorname{grad}\left(\psi^{(2)}-\psi_{h}^{(2)}\right)\right.}\right)_{\Gamma^{(1)}} \\
& +\sum_{T \in \mathcal{M}_{h}}\left\{\int_{T} \omega^{2} \hat{\varepsilon} \widetilde{\mathbf{E}}_{h}^{\mathrm{PML}} \cdot \overline{\left(\psi^{(1)}-\psi_{h}^{(1)}\right)}+\int_{T} \omega^{2} \hat{\varepsilon} \widetilde{\mathbf{E}}_{h}^{\mathrm{PML}} \cdot \operatorname{grad} \overline{\left(\psi^{(2)}-\psi_{h}^{(2)}\right)}\right. \\
& \left.-\int_{T} \hat{\mu}^{-1} \operatorname{curl} \widetilde{\mathbf{E}}_{h}^{\mathrm{PML}} \cdot \operatorname{curl} \overline{\left(\psi^{(1)}-\psi_{h}^{(1)}\right)}\right\} .
\end{aligned}
$$


A direct calculation and (3.2) yield

$$
\begin{aligned}
& \left.\int_{\Gamma^{(1)}} \frac{1}{\mu^{(1)}} \operatorname{curl}\left(\mathbf{E}_{\mathrm{I}}-\mathbf{E}_{\mathrm{I}}^{\mathbf{r}}\right) \times \nu_{3} \cdot \overline{\left(\operatorname{grad}\left(\psi^{(2)}-\psi_{h}^{(2)}\right)\right.}\right)_{\Gamma^{(1)}} \\
& \left.=-\int_{\Gamma^{(1)}} \frac{1}{\mu^{(1)}} \operatorname{curl} \operatorname{curl}\left(\mathbf{E}_{\mathrm{I}}-\mathbf{E}_{\mathrm{I}}^{\mathbf{r}}\right) \cdot \nu_{3} \overline{\left(\psi^{(2)}-\psi_{h}^{(2)}\right.}\right) \\
& =-\int_{\Gamma^{(1)}} \omega^{2} \varepsilon\left(\mathbf{E}_{\mathrm{I}}-\mathbf{E}_{\mathrm{I}}^{\mathbf{r}}\right) \cdot \nu_{3}\left(\overline{\psi^{(2)}-\psi_{h}^{(2)}}\right) .
\end{aligned}
$$

Therefore by integration by parts and using (3.8)-(3.11),

$$
\begin{aligned}
\mathrm{II}+\mathrm{III}= & \left.\sum_{F \in \mathcal{F}_{h}, F \subset \Gamma^{(1)}} \int_{F} \frac{1}{\mu^{(1)}} \operatorname{curl}\left(\mathbf{E}_{\mathrm{I}}-\mathbf{E}_{\mathrm{I}}^{\mathbf{r}}\right) \times \nu_{3} \cdot \overline{\left(\psi^{(1)}-\psi_{h}^{(1)}\right.}\right)_{\Gamma^{(1)}} \\
& -\sum_{F \in \mathcal{F}_{h}, F \subset \Gamma^{(1)}} \int_{F} \omega^{2} \varepsilon\left(\mathbf{E}_{\mathrm{I}}-\mathbf{E}_{\mathrm{I}}^{\mathbf{r}}\right) \cdot \nu_{3}\left(\overline{\psi^{(2)}-\psi_{h}^{(2)}}\right) \\
& +\sum_{T \in \mathcal{M}_{h}}\left\{\int_{T}\left(\omega^{2} \hat{\varepsilon} \widetilde{\mathbf{E}}_{h}^{\mathrm{PML}}-\operatorname{curl}\left(\hat{\mu}^{-1} \operatorname{curl} \widetilde{\mathbf{E}}_{h}^{\mathrm{PML}}\right)\right) \cdot \overline{\left(\psi^{(1)}-\psi_{h}^{(1)}\right.}\right) \\
& \left.-\int_{T} \operatorname{div}\left(\omega^{2} \hat{\varepsilon} \widetilde{\mathbf{E}}_{h}^{\mathrm{PML}}\right) \cdot \overline{\left(\psi^{(2)}-\psi_{h}^{(2)}\right.}\right) \\
& +\sum_{F \in \mathcal{F}_{h}, F \subset \partial T}\left[-\int_{F} \hat{\mu}^{-1} \operatorname{curl} \widetilde{\mathbf{E}}_{h}^{\mathrm{PML}} \times \nu \cdot \overline{\psi^{(1)}-\psi_{h}^{(1)}}\right)_{F} \\
= & \left.\left.\sum_{T \in \mathcal{M}_{h}}\left\{\int_{T} R_{T}^{(1)} \cdot \overline{\left(\psi^{(1)}-\psi_{h}^{(1)}\right.}\right)+\int_{T} \hat{R}_{T}^{(2)} \overline{\left(\psi^{(2)}-\psi_{h}^{(2)}\right.}\right)\right\} \\
& \left.\left.+\sum_{F \in \mathcal{F}_{h}, F \not \subset \Gamma_{11} \cup \Gamma_{21}}\left\{\int_{F} J_{F}^{(1)} \cdot \overline{\left(\psi^{(2)}-\psi_{h}^{(2)}\right.}\right)\right]\right\}
\end{aligned}
$$

Here $\left(\psi^{(1)}-\psi_{h}^{(1)}\right)_{F}=-\nu \times\left(\nu \times\left(\psi^{(1)}-\psi_{h}^{(1)}\right)\right)$ is the tangential component of $\psi^{(1)}-$ $\psi_{h}^{(1)}$ along $F$. It follows from (4.6), (4.8)-(4.9), the Cauchy-Schwarz inequality, and (4.4) that

$$
\begin{aligned}
\mid \mathrm{II} & +\mathrm{III} \mid \\
\leq & \sum_{T \in \mathcal{M}_{h}}\left\{C h_{T}\left\|R_{T}^{(1)}\right\|_{\left(L^{2}(T)\right)^{3}}\left|\psi^{(1)}\right|_{\left(H^{1}(\widetilde{T})\right)^{3}}+C h_{T}\left\|R_{T}^{(2)}\right\|_{L^{2}(T)}\left|\psi^{(2)}\right|_{H^{1}(\widetilde{T})}\right\} \\
& +\sum_{F \in \mathcal{F}_{h}}\left\{C h_{F}^{1 / 2}\left\|J_{F}^{(1)}\right\|_{\left(L^{2}(F)\right)^{3}}\left|\psi^{(1)}\right|_{\left(H^{1}(\widetilde{F})\right)^{3}}+C h_{F}^{1 / 2}\left\|J_{F}^{(2)}\right\|_{L^{2}(F)}\left|\psi^{(2)}\right|_{H^{1}(\widetilde{F})}\right\} \\
\leq & C\left(\sum_{T \in \mathcal{M}_{h}} \eta_{T}^{2}\right)^{1 / 2}\left(\left|\psi^{(1)}\right|_{\left(H^{1}(D)\right)^{3}}^{2}+\left|\psi^{(2)}\right|_{H^{1}(D)}^{2}\right)^{1 / 2} \\
\leq & C\left(\sum_{T \in \mathcal{M}_{h}} \eta_{T}^{2}\right)^{1 / 2}\|\psi\|_{H(\operatorname{curl}, D)} .
\end{aligned}
$$


From Lemma 4.3 we obtain

$$
|\mathrm{II}+\mathrm{III}| \leq C\left[1+\gamma_{0} \max \left|s\left(x_{3}\right)\right|\left(C^{(1)}+C^{(2)}\right)\right]\left(\sum_{T \in \mathcal{M}_{h}} \eta_{T}^{2}\right)^{1 / 2}\|\psi\|_{H(\operatorname{curl}, \Omega)} .
$$

Secondly, the estimate of $|\mathrm{IV}+\mathrm{V}|$ follows from Lemmas 2.5 and 2.2

$$
|\mathrm{IV}+\mathrm{V}| \leq \gamma_{0}^{2}\left(M^{(1)}\left\|\widetilde{\mathbf{E}}_{h}^{\mathrm{PML}}-\mathbf{E}_{\mathrm{I}}\right\|_{H(\operatorname{curl}, \Omega)}+M^{(2)}\left\|\widetilde{\mathbf{E}}_{h}^{\mathrm{PML}}\right\|_{H(\operatorname{curl}, \Omega)}\right)\|\psi\|_{H(\operatorname{curl}, \Omega)} .
$$

Combining (4.12) and (4.13) yields the desired estimate.

\section{IMPLEMENTATION AND NUMERICAL EXAMPLES}

The implementation of the adaptive algorithm is based on the Comsol Multiphysics software package. The second-order Nédélec's edge element is used in the numerical tests. Numerically, we consider nonmagnetic materials, i.e., $\mu(x)=I$ and $\mu^{(1)}=\mu^{(2)}=1$. We use the a posteriori error estimate from Theorem 3.1 to determine the PML parameters. Recall that we choose the PML medium property as the power function, and thus we need to specify only the thickness $\delta_{j}$ of the layers and the medium parameters $\sigma_{j}^{m}$ (see (2.531). Theorem 3.1 shows that the $a$ posteriori error estimate consists of two parts: the PML error $\mathcal{E}_{\mathrm{PML}}$ and the finite element discretization error $\mathcal{E}_{\mathrm{FEM}}$, where

$$
\begin{aligned}
& \mathcal{E}_{\mathrm{PML}}=\gamma_{0}^{2} M^{(1)}\left\|\widetilde{\mathbf{E}}_{h}^{\mathrm{PML}}-\mathbf{E}_{\mathrm{I}}\right\|_{H(\operatorname{curl}, \Omega)}+\gamma_{0}^{2} M^{(2)}\left\|\widetilde{\mathbf{E}}_{h}^{\mathrm{PML}}\right\|_{H(\mathrm{curl}, \Omega)}, \\
& \mathcal{E}_{\mathrm{FEM}}=\left(\sum_{T \in \mathcal{M}_{h}} \eta_{T}^{2}\right)^{1 / 2} .
\end{aligned}
$$

Notice from the definition of $M^{(j)}$ in Lemma 2.5 that $M^{(j)} /\left(2 /\left(e^{\Lambda^{(j)}}-1\right)\right)$ is bounded. In practice, we first choose $\delta_{j}$ and $\sigma_{j}^{m}$ such that $2 /\left(e^{\Lambda^{(j)}}-1\right) \leq 10^{-8}$, which makes the PML error negligible compared with the finite element discretization errors. Once the PML region and the medium property are fixed, we use the standard finite element adaptive strategy to modify the mesh according to the a posteriori error estimate (5.2). The adaptive algorithm used in this paper is described as follows:

Algorithm 5.1. Given tolerance TOL $>0$. Let $m=2$.

- Choose $\delta_{1}, \delta_{2}$, and $\sigma_{j}^{m}$ such that $2 /\left(e^{\Lambda^{(j)}}-1\right) \leq 10^{-8}(j=1,2)$.

- Generate an initial mesh $\mathcal{M}_{h}$ over D.

- While $\mathcal{E}_{\mathrm{FEM}}>\mathrm{TOL} d o$

- Choose a set of elements $\widehat{\mathcal{M}}_{h} \subset \mathcal{M}_{h}$ such that

$$
\left(\sum_{T \in \widehat{\mathcal{M}}_{h}} \eta_{T}^{2}\right)^{1 / 2}>0.6\left(\sum_{T \in \mathcal{M}_{h}} \eta_{T}^{2}\right)^{1 / 2} ;
$$

then refine the elements in $\widehat{\mathcal{M}}_{h}$. Denote the new mesh by $\mathcal{M}_{h}$ also.

- Solve the discrete problem (3.7) on $\mathcal{M}_{h}$.

- Compute error estimators on $\mathcal{M}_{h}$. end while 
We remark that, due to the periodic structure of the grating problem, we modified the local tetrahedral mesh refinement algorithm by Arnold, Mukherjee, and Pouly [4] to maintain the periodicity of the meshes in both the $x_{1}$ and $x_{2}$ directions, which either the algorithm of COMSOL Multiphysics or the algorithm of [4] does not take into account.

We report two examples to demonstrate the competitiveness of our algorithm. In all of the experiments, let $\lambda, \theta_{1}, \theta_{2}$, and $p=\left(p_{1}, p_{2}, p_{3}\right)^{\mathrm{T}}$ denote the wavelength, the incident angles, and the polarization of the incident wave, respectively, and let $n$ denote the refractive index. We scale the error estimator by a factor of $6 \times 10^{-3}$ in the following examples.

Example 5.1. To test our code, we consider the simplest grating structure, a flat plane. Assume that a plane wave $\left(\mathbf{E}_{\mathrm{I}}, \mathbf{H}_{\mathrm{I}}\right)$ is incident on the flat plane $\left\{x_{3}=0\right\}$, which separates two homogeneous media: vacuum $\left(n_{1}=1\right)$ and silver $\left(n_{2}=0.22+\right.$ 6.71i). In our experiment, the parameters are chosen as $\lambda=1 \mu \mathrm{m}, \theta_{1}=\pi / 6, \theta_{2}=$ $\pi / 6, p_{1}=p_{2}=1$. For this problem, the exact zero-order reflection efficiency is 0.9784459 . The computation domain is chosen as $\Omega=[0,0.5] \times[0,0.5] \times[-0.25,0.25]$.

The mesh plot and the surface plot of the amplitude of the field $\widetilde{\mathbf{E}}_{h}^{\text {PML }}$ after 13 adaptive iterations are shown in Figure 2. The mesh has 36118 tetrahedrons and the total number of degrees of freedom (DoFs) on the mesh is 235476. Figure 3 shows the error of $\widetilde{\mathbf{E}}_{h}^{\text {PML }}$ in the $H(\operatorname{curl}, \Omega)$ norm (left: solid line), the a posteriori error estimate (left: circles), and the error of the zero-order reflection efficiencies (right) as a function of the total number of DoFs. It is shown that the decay of $\left\|\widetilde{\mathbf{E}}_{h}^{\mathrm{PML}}-\mathbf{E}\right\|_{H(\operatorname{curl}, \Omega)}$ is $O\left(1 / N^{2 / 3}\right)$ and the convergence rate of the zero-order reflection efficiencies is about $O\left(1 / N^{4 / 3}\right)$, where $N$ is the total number of DoFs.
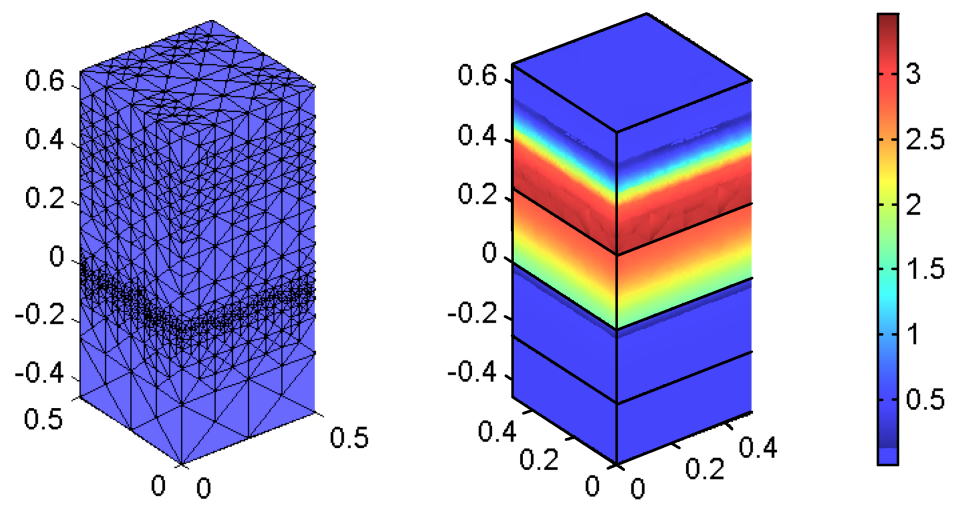

Figure 2. The mesh plot and the surface plot of the amplitude of the field $\widetilde{\mathbf{E}}_{h}^{\text {PML }}$ after 13 adaptive iterations for Example [5.1.

Example 5.2. Consider the checkerboard grating that was analyzed by $\mathrm{Li} 33$ using a new Fourier modal method. A top view of it along with the unit cell and the computational domain are shown in Figure 4. The length, width, and height of the shadowed cubic are $1.25 \mu \mathrm{m}, 1.25 \mu \mathrm{m}$, and $1 \mu \mathrm{m}$. The grating parameters are: $\lambda=1 \mu \mathrm{m}, \theta_{1}=0, \theta_{2}=0, p_{1}=p_{2}=1$, the refractive indices of the shadowed cubic and the superstrate are 1.5, and the refractive index of the other part is 1 . 

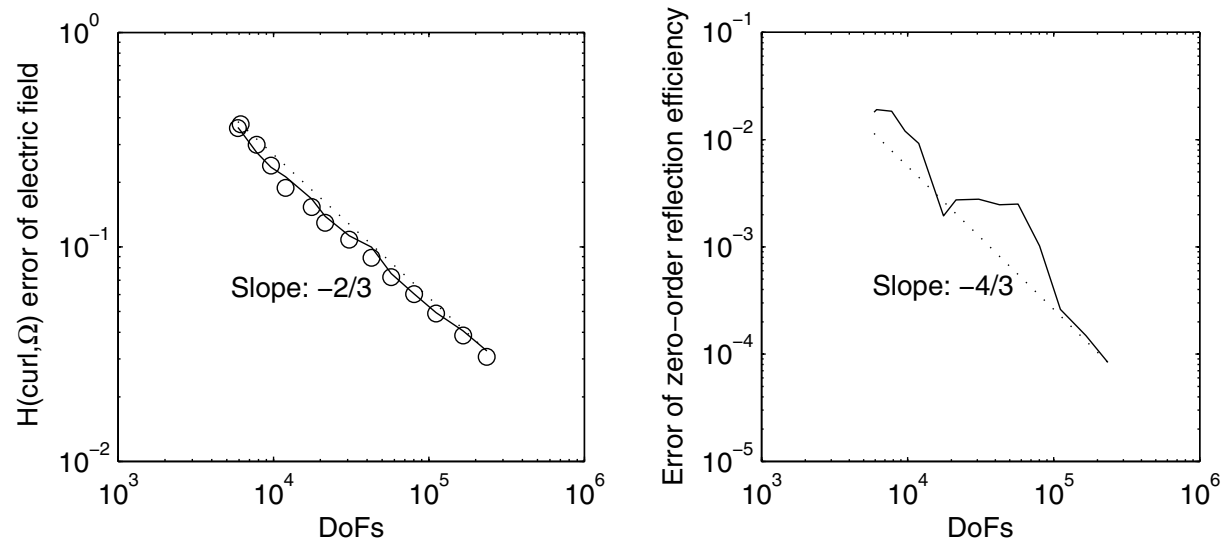

FiguRE 3. The error of $\widetilde{\mathbf{E}}_{h}^{\mathrm{PML}}$ in the $H(\operatorname{curl}, \Omega)$ norm (left: solid line) and the a posteriori error estimate (left: circles), and the error of the zero-order reflection efficiencies (right) versus the total number of degrees of freedom for Example 5.1. The dotted line gives the reference slope.
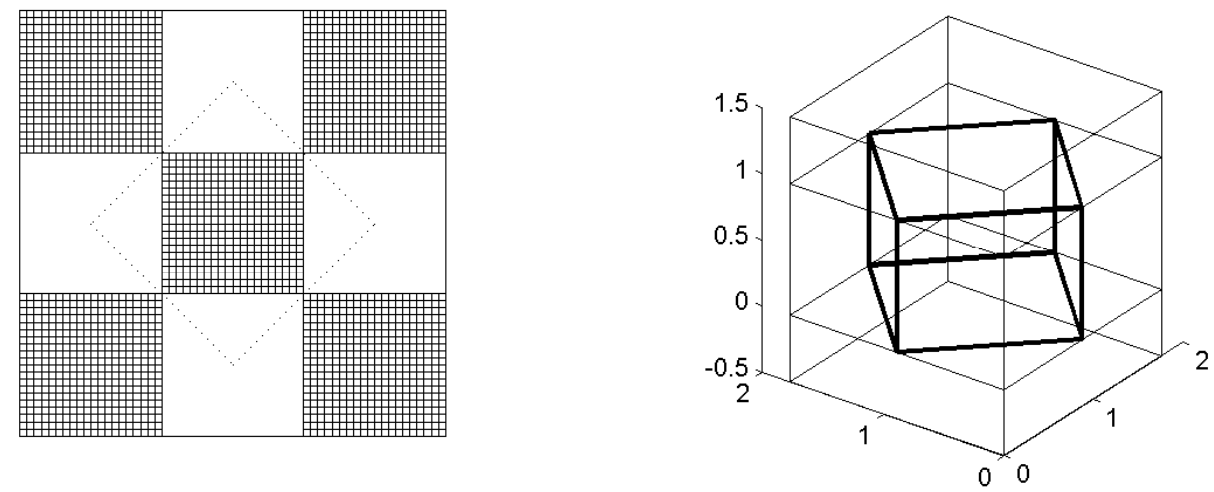

Figure 4. A top view of the grating along with the unit cell (left), and the computational domain (right) for Example 5.2

Figure 5 shows that the efficiencies are convergent (left) and that the decay of the a posteriori error estimates is $O\left(1 / N^{2 / 3}\right)$ (right). The mesh plot and the surface plot of the amplitude of the field $\widetilde{\mathbf{E}}_{h}^{\text {PML }}$ after 10 adaptive iterations are shown in Figure 6. The mesh has 60042 tetrahedrons and the total number of DoFs on the mesh is 391244 . The meshes near the upper PML boundary are rather coarse, which shows that the total computational cost is insensitive to the thickness of the PML absorbing layer. Recall that thicker PML layers allow a smaller PML medium property, which enhances numerical stability. Table 1 shows the diffraction efficiencies of the transmitted orders of the checkerboard grating. The maximum error between our results and those of $\mathrm{Li} 33$ is about $8.13 \times 10^{-4}$. 

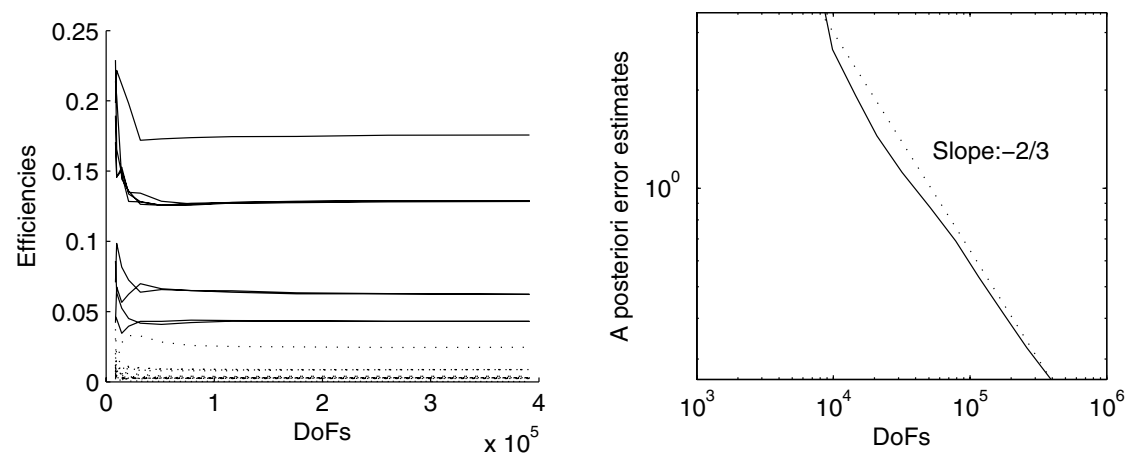

Figure 5. Transmission efficiencies (solid lines) and reflection efficiencies (dotted lines) versus the total number of DoFs (left), and the a posteriori error estimates versus the total number of DoFs (right) for Example 5.2.
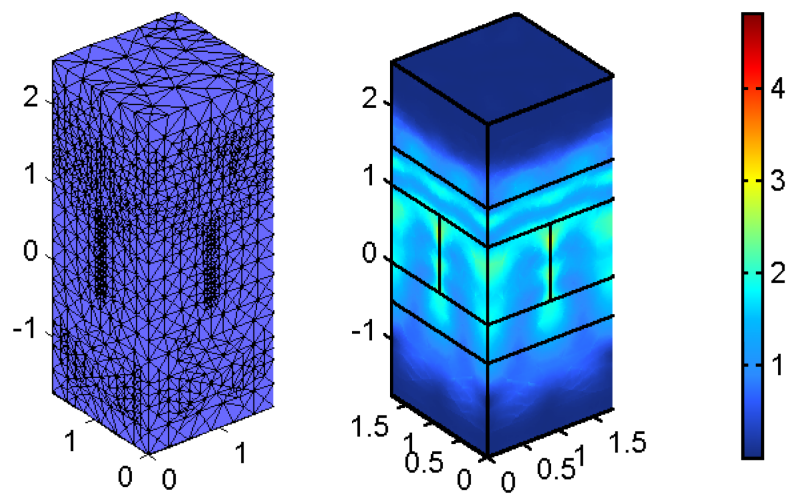

Figure 6 . The mesh plot and the surface plot of the amplitude of the field $\widetilde{\mathbf{E}}_{h}^{\text {PML }}$ after 10 adaptive iterations for Example 5.2.

TABLE 1. Diffraction efficiencies of the transmitted orders of the checkerboard grating in Example 5.2 .

\begin{tabular}{cccc}
\hline & & & \\
Diffraction order & -1 & 0 & 1 \\
\hline-1 & 0.0431 & 0.1287 & 0.0623 \\
0 & 0.1284 & 0.1757 & 0.1288 \\
1 & 0.0622 & 0.1287 & 0.0430 \\
\hline
\end{tabular}

Appendix A. Proofs of technical lemmas

A.1. Proof of Lemma 4.3, By a similar argument for (2.35), $\psi$ satisfies the following expansion in $\Omega^{(j, \mathrm{PML})}$ :

$$
\bar{\psi}=\operatorname{diag}\left(1,1, s\left(x_{3}\right)\right) \sum_{l, m \in Z}\left(\left(p_{l, m}^{(j)}\right)^{\prime} e^{-\mathbf{i}\left(q_{l, m}^{(j)}\right)^{\prime} \cdot \hat{x}}-\zeta_{l, m}^{(j)} p_{l, m}^{(j)} e^{-\mathbf{i} q_{l, m}^{(j)} \cdot \hat{x}}\right)^{\mathrm{T}},
$$


where $q_{l, m}^{(j)}=\left(\alpha_{1}^{l}, \alpha_{2}^{m},(-1)^{j-1} \beta_{l, m}^{(j)}\right)^{\mathrm{T}}$ is defined in (2.12), $p_{l, m}^{(j)}=\left(p_{l, m, 1}^{(j)}, p_{l, m, 2}^{(j)}\right.$, $\left.p_{l, m, 3}^{(j)}\right)^{\mathrm{T}}$ satisfies $p_{l, m}^{(j)} \cdot q_{l, m}^{(j)}=0$, and

$$
\zeta_{l, m}^{(j)}=e^{2 \mathbf{i}(-1)^{j-1} \beta_{l, m}^{(j)} \int_{0}^{b^{(1)}+(-1)^{j-1} \delta^{(j)}} s(\tau) \mathrm{d} \tau}
$$

is defined in (2.36). Denote by

$$
\xi_{l, m}^{j \pm}\left(x_{3}\right)= \pm e^{\mathbf{i}(-1)^{j-1} \beta_{l, m}^{(j)} \int_{0}^{x_{3}} s(\tau) d \tau}-\zeta_{l, m}^{(j)} e^{-\mathbf{i}(-1)^{j-1} \beta_{l, m}^{(j)} \int_{0}^{x_{3}} s(\tau) \mathrm{d} \tau} .
$$

Then $\bar{\psi}$ can be rewritten as

$$
\bar{\psi}=\sum_{l, m \in Z}\left(p_{l, m, 1}^{(j)} \xi_{l, m}^{j+}\left(x_{3}\right), p_{l, m, 2}^{(j)} \xi_{l, m}^{j+}\left(x_{3}\right), s\left(x_{3}\right) p_{l, m, 3}^{(j)} \xi_{l, m}^{j-}\left(x_{3}\right)\right)^{\mathrm{T}} e^{-\mathbf{i} \alpha_{1}^{l} x_{1}-\mathbf{i} \alpha_{2}^{m} x_{2}} .
$$

It is clear that

$$
\frac{\mathrm{d}}{\mathrm{d} x_{3}} \xi_{l, m}^{j \pm}\left(x_{3}\right)=\mathbf{i}(-1)^{j} \beta_{l, m}^{(j)} s\left(x_{3}\right) \xi_{l, m}^{j \mp}\left(x_{3}\right) .
$$

Therefore

$$
\operatorname{curl} \bar{\psi}=\sum_{l, m \in Z}\left(\begin{array}{c}
-\left[\mathbf{i} \alpha_{2}^{m} p_{l, m, 3}^{(j)}+\mathbf{i}(-1)^{j} \beta_{l, m}^{(j)} p_{l, m, 2}^{(j)}\right] s\left(x_{3}\right) \xi_{l, m}^{j-}\left(x_{3}\right) \\
{\left[\mathbf{i} \alpha_{1}^{l} p_{l, m, 3}^{(j)}+\mathbf{i}(-1)^{j} \beta_{l, m}^{(j)} p_{l, m, 1}^{(j)}\right] s\left(x_{3}\right) \xi_{l, m}^{j-}\left(x_{3}\right)} \\
{\left[-\mathbf{i} \alpha_{1}^{l} p_{l, m, 2}^{(j)}+\mathbf{i} \alpha_{2}^{m} p_{l, m, 1}^{(j)}\right] \xi_{l, m}^{j+}\left(x_{3}\right)}
\end{array}\right) e^{-\mathbf{i} \alpha_{1}^{l} x_{1}-\mathbf{i} \alpha_{2}^{m} x_{2}}
$$

Let $I^{(1)}=\left[b^{(1)}, b^{(1)}+\delta^{(1)}\right]$ and $I^{(2)}=\left[b^{(2)}-\delta^{(2)}, b^{(2)}\right]$. From a direct calculation, we deduce from (A.3) and (A.4) that

$$
\begin{aligned}
& \left\|s^{-1} \psi\right\|_{\left(L^{2}\left(\Omega^{(j, \mathrm{PML})}\right)\right)^{3}}^{2}+\left\|s^{-1} \operatorname{curl} \psi\right\|_{\left(L^{2}\left(\Omega^{(j, \mathrm{PML})}\right)\right)^{3}}^{2} \\
& =\int_{I^{(j)}}\left|s\left(x_{3}\right)\right|^{-2} \int_{0}^{L_{1}} \int_{0}^{L_{2}}\left(|\bar{\psi}|^{2}+|\operatorname{curl} \bar{\psi}|^{2}\right) \mathrm{d} x_{1} \mathrm{~d} x_{2} \mathrm{~d} x_{3} \\
& =L_{1} L_{2} \int_{I^{(j)}}\left|s\left(x_{3}\right)\right|^{-2} \sum_{l, m \in Z}\left[\left|p_{l, m, 1}^{(j)}\right|^{2}\left|\xi_{l, m}^{j+}\left(x_{3}\right)\right|^{2}+\left|p_{l, m, 2}^{(j)}\right|^{2}\left|\xi_{l, m}^{j+}\left(x_{3}\right)\right|^{2}\right. \\
& \quad+\left|s\left(x_{3}\right)\right|^{2}\left|p_{l, m, 3}^{(j)}\right|^{2}\left|\xi_{l, m}^{j-}\left(x_{3}\right)\right|^{2}+\left|\alpha_{1}^{l} p_{l, m, 2}^{(j)}-\alpha_{2}^{m} p_{l, m, 1}^{(j)}\right|^{2}\left|\xi_{l, m}^{j+}\left(x_{3}\right)\right|^{2} \\
& \quad+\left|\alpha_{2}^{m} p_{l, m, 3}^{(j)}+(-1)^{j} \beta_{l, m}^{(j)} p_{l, m, 2}^{(j)}\right|^{2}\left|s\left(x_{3}\right)\right|^{2}\left|\xi_{l, m}^{j-}\left(x_{3}\right)\right|^{2} \\
& \left.\quad+\left|\alpha_{1}^{l} p_{l, m, 3}^{(j)}+(-1)^{j} \beta_{l, m}^{(j)} p_{l, m, 1}^{(j)}\right|^{2}\left|s\left(x_{3}\right)\right|^{2}\left|\xi_{l, m}^{j-}\left(x_{3}\right)\right|^{2}\right] \mathrm{d} x_{3} .
\end{aligned}
$$

Since $\left|s\left(x_{3}\right)\right|^{-1} \leq 1$, we have

$$
\begin{aligned}
& \left\|s^{-1} \bar{\psi}\right\|_{\left(L^{2}\left(\Omega^{(j, \mathrm{PML})}\right)\right)^{3}}^{2}+\left\|s^{-1} \operatorname{curl} \bar{\psi}\right\|_{\left(L^{2}\left(\Omega^{(j, \mathrm{PML})}\right)\right)^{3}}^{2} \\
& \leq L_{1} L_{2} \int_{I^{(j)}} \sum_{l, m \in Z}\left[\left(\left|p_{l, m, 1}^{(j)}\right|^{2}+\left|p_{l, m, 2}^{(j)}\right|^{2}+\left|\alpha_{1}^{l} p_{l, m, 2}^{(j)}-\alpha_{2}^{m} p_{l, m, 1}^{(j)}\right|^{2}\right)\left|\xi_{l, m}^{j+}\left(x_{3}\right)\right|^{2}\right. \\
& +\left(\left|\alpha_{2}^{m} p_{l, m, 3}^{(j)}+(-1)^{j} \beta_{l, m}^{(j)} p_{l, m, 2}^{(j)}\right|^{2}\right. \\
& +\left|\alpha_{1}^{l} p_{l, m, 3}^{(j)}+(-1)^{j} \beta_{l, m}^{(j)} p_{l, m, 1}^{(j)}\right|^{2} \\
& \left.\left.+\left|p_{l, m, 3}^{(j)}\right|^{2}\right)\left|\xi_{l, m}^{j-}\left(x_{3}\right)\right|^{2}\right] \mathrm{d} x_{3} .
\end{aligned}
$$


On the other hand, from (A.3), the tangential component of $\bar{\psi}$ on the surface $\Gamma^{(j)}$ is

$$
\bar{\psi}_{\Gamma^{(j)}}=\sum_{l, m \in Z}\left(p_{l, m, 1}^{(j)} \xi_{l, m}^{j+}\left(b^{(j)}\right), p_{l, m, 2}^{(j)} \xi_{l, m}^{j+}\left(b^{(j)}\right), 0\right)^{\mathrm{T}} e^{-\mathbf{i} \alpha_{1}^{l} x_{1}-\mathbf{i} \alpha_{2}^{m} x_{2}}
$$

Hence, it is follows from (2.7) that

$$
\begin{aligned}
\left\|\bar{\psi}_{\Gamma^{(j)}}\right\|_{T H_{\text {qper }}^{-1 / 2}\left(\operatorname{curl}, \Gamma^{(j)}\right)}^{2}= & L_{1} L_{2} \sum_{l, m \in Z}\left(1+\left(\alpha_{1}^{l}\right)^{2}+\left(\alpha_{2}^{m}\right)^{2}\right)^{-1 / 2}\left|\xi_{l, m}^{j+}\left(b^{(j)}\right)\right|^{2} \\
& \times\left[\left|p_{l, m, 1}^{(j)}\right|^{2}+\left|p_{l, m, 2}^{(j)}\right|^{2}+\left|\alpha_{1}^{l} p_{l, m, 2}^{(j)}-\alpha_{2}^{m} p_{l, m, 1}^{(j)}\right|^{2}\right] .
\end{aligned}
$$

From Lemma 2.2, it suffices to prove that

$$
\begin{aligned}
& \left\|s^{-1} \bar{\psi}\right\|_{\left(L^{2}\left(\Omega^{(j, \mathrm{PML})}\right)\right)^{3}}^{2}+\left\|s^{-1} \operatorname{curl} \bar{\psi}\right\|_{\left(L^{2}\left(\Omega^{(j, \mathrm{PML})}\right)\right)^{3}}^{2} \\
& \leq\left(C^{(j)}\right)^{2}\left\|\bar{\psi}_{\Gamma^{(j)}}\right\|_{T H_{\mathrm{qper}}^{-1 / 2}\left(\operatorname{curl}, \Gamma^{(j)}\right)}^{2} .
\end{aligned}
$$

To this end, we will estimate the terms on the right-hand side of A.5.

Since $p_{l, m}^{(j)} \cdot q_{l, m}^{(j)}=0$, we have

$$
p_{l, m, 3}^{(j)}=\frac{(-1)^{j}}{\beta_{l, m}^{(j)}}\left(\alpha_{1}^{l} p_{l, m, 1}^{(j)}+\alpha_{2}^{m} p_{l, m, 2}^{(j)}\right)
$$

and

$$
\left|p_{l, m, 3}^{(j)}\right|^{2} \leq \frac{1}{\left|\beta_{l, m}^{(j)}\right|^{2}}\left(\left(\alpha_{1}^{l}\right)^{2}+\left(\alpha_{2}^{m}\right)^{2}\right)\left(\left|p_{l, m, 1}^{(j)}\right|^{2}+\left|p_{l, m, 2}^{(j)}\right|^{2}\right) .
$$

From (A.8) and the definition $\left(\beta_{l, m}^{(j)}\right)^{2}=\omega^{2} \varepsilon^{(j)} \mu^{(j)}-\left(\alpha_{1}^{l}\right)^{2}-\left(\alpha_{2}^{m}\right)^{2}$ in (2.13), we conclude that

$$
\begin{aligned}
\alpha_{2}^{m} p_{l, m, 3}^{(j)}+(-1)^{j} \beta_{l, m}^{(j)} p_{l, m, 2}^{(j)} & =\frac{(-1)^{j}}{\beta_{l, m}^{(j)}}\left[\omega^{2} \varepsilon^{(j)} \mu^{(j)} p_{l, m, 2}^{(j)}-\alpha_{1}^{l}\left(\alpha_{1}^{l} p_{l, m, 2}^{(j)}-\alpha_{2}^{m} p_{l, m, 1}^{(j)}\right)\right] \\
\alpha_{1}^{l} p_{l, m, 3}^{(j)}+(-1)^{j} \beta_{l, m}^{(j)} p_{l, m, 1}^{(j)} & =\frac{(-1)^{j}}{\beta_{l, m}^{(j)}}\left[\omega^{2} \varepsilon^{(j)} \mu^{(j)} p_{l, m, 1}^{(j)}+\alpha_{2}^{m}\left(\alpha_{1}^{l} p_{l, m, 2}^{(j)}-\alpha_{2}^{m} p_{l, m, 1}^{(j)}\right)\right] .
\end{aligned}
$$

From the identity $|a+b|^{2}=|a|^{2}+|b|^{2}+2 \operatorname{Re}(\bar{a} b)$, the fact that $\operatorname{Re}\left(\omega^{2} \varepsilon^{(j)} \mu^{(j)}\right)>0$, and some direct calculations, we have

$$
\begin{gathered}
\left|\alpha_{2}^{m} p_{l, m, 3}^{(j)}+(-1)^{j} \beta_{l, m}^{(j)} p_{l, m, 2}^{(j)}\right|^{2}+\left|\alpha_{1}^{l} p_{l, m, 3}^{(j)}+(-1)^{j} \beta_{l, m}^{(j)} p_{l, m, 1}^{(j)}\right|^{2} \\
\leq \frac{1}{\left|\beta_{l, m}^{(j)}\right|^{2}}\left[\left|\omega^{2} \varepsilon^{(j)} \mu^{(j)}\right|^{2}\left(\left|p_{l, m, 1}^{(j)}\right|^{2}+\left|p_{l, m, 2}^{(j)}\right|^{2}\right)\right. \\
\left.+\left(\left(\alpha_{1}^{l}\right)^{2}+\left(\alpha_{2}^{m}\right)^{2}\right)\left|\alpha_{1}^{l} p_{l, m, 2}^{(j)}-\alpha_{2}^{m} p_{l, m, 1}^{(j)}\right|^{2}\right] .
\end{gathered}
$$


Next we estimate $\int_{I^{(j)}}\left|\xi_{l, m}^{j \pm}\left(x_{3}\right)\right|^{2} \mathrm{~d} x_{3}$. From (A.2) and Lemma 2.4 we conclude that

$$
\begin{aligned}
\left|\xi_{l, m}^{j \pm}\left(x_{3}\right)\right| & =\left|e^{\mathbf{i}(-1)^{j-1} \beta_{l, m}^{(j)} \int_{0}^{x_{3}} s(\tau) \mathrm{d} \tau}\right| \cdot\left| \pm 1-e^{2 \mathbf{i}(-1)^{j-1} \beta_{l, m}^{(j)} \int_{x_{3}}^{b^{(j)}+(-1)^{j-1} \delta^{(j)}} s(\tau) \mathrm{d} \tau}\right| \\
& \leq 2\left|e^{\mathbf{i}(-1)^{j-1} \beta_{l, m}^{(j)} \int_{0}^{x_{3}} s(\tau) \mathrm{d} \tau}\right| \\
\left|\xi_{l, m}^{j+}\left(b^{(j)}\right)\right| & \geq\left|e^{\mathbf{i}(-1)^{j-1} \beta_{l, m}^{(j)} \int_{0}^{b^{(j)}} s(\tau) \mathrm{d} \tau}\right|\left(1-\left|e^{2 \mathbf{i}(-1)^{j-1} \beta_{l, m}^{(j)} \int_{b}^{b(j)}+(-1)^{j-1} \delta^{(j)}} s(\tau) \mathrm{d} \tau\right|\right) \\
& \geq\left|e^{\mathbf{i}(-1)^{j-1} \beta_{l, m}^{(j)} \int_{0}^{b(j)} s(\tau) \mathrm{d} \tau}\right|\left(1-e^{-\Lambda^{(j)}}\right) .
\end{aligned}
$$

Here $\Lambda^{(j)}$ is defined in Lemma 2.5. Therefore

$$
\begin{aligned}
\left|\xi_{l, m}^{j \pm}\left(x_{3}\right)\right| /\left|\xi_{l, m}^{j+}\left(b^{(j)}\right)\right| & \leq \frac{2}{1-e^{-\Lambda^{(j)}}}\left|e^{\mathbf{i}(-1)^{j-1} \beta_{l, m}^{(j)} \int_{b}^{x_{3}} s(\tau) \mathrm{d} \tau}\right| \\
& =\frac{2}{1-e^{-\Lambda^{(j)}}} \times e^{(-1)^{j-1} \int_{b(j)}^{x_{3}} \operatorname{Re}\left(\mathbf{i} \beta_{l, m}^{(j)} s(\tau)\right) \mathrm{d} \tau} \\
& \leq \frac{2}{1-e^{-\Lambda^{(j)}}} \times e^{-(-1)^{j-1} \int_{b}^{x_{3}} \operatorname{Im}\left(\beta_{l, m}^{(j)}\right) s_{1}(\tau) \mathrm{d} \tau} \\
& \leq \frac{2}{1-e^{-\Lambda^{(j)}}} \times e^{-(-1)^{j-1} \operatorname{Im}\left(\beta_{l, m}^{(j)}\right)\left(x_{3}-b^{(j)}\right)} .
\end{aligned}
$$

Here we have used $s_{1}(\tau) \geq 1$ to derive the last inequality. Thus

(A.11)

$$
\int_{I^{(j)}}\left|\xi_{l, m}^{j \pm}\left(x_{3}\right)\right|^{2} \mathrm{~d} x_{3} /\left|\xi_{l, m}^{j+}\left(b^{(j)}\right)\right|^{2} \leq \frac{4}{\left(1-e^{\left.-\Lambda^{(j)}\right)^{2}}\right.} \min \left\{\delta^{(j)}, \frac{1}{2 \operatorname{Im}\left(\beta_{l, m}^{(j)}\right)}\right\} .
$$

Combining the estimates (A.5) and (A.9) - A.11), we attain that

$$
\begin{aligned}
& \left\|s^{-1} \bar{\psi}\right\|_{\left(L^{2}\left(\Omega^{(j, \mathrm{PML})}\right)\right)^{3}}^{2}+\left\|s^{-1} \operatorname{curl} \bar{\psi}\right\|_{\left(L^{2}\left(\Omega^{(j, \mathrm{PML})}\right)\right)^{3}}^{2} \\
& \leq L_{1} L_{2} \sum_{l, m \in Z}\left(1+\frac{\left(\alpha_{1}^{l}\right)^{2}+\left(\alpha_{2}^{m}\right)^{2}+\left|\omega^{2} \varepsilon^{(j)} \mu^{(j)}\right|^{2}}{\left|\beta_{l, m}^{(j)}\right|^{2}}\right) \min \left\{2 \delta^{(j)}, \frac{1}{\operatorname{Im}\left(\beta_{l, m}^{(j)}\right)}\right\} \\
& \quad \times \frac{2}{\left(1-e^{-\Lambda^{(j)}}\right)^{2}}\left|\xi_{l, m}^{j+}\left(b^{(j)}\right)\right|^{2}\left(\left|p_{l, m, 1}^{(j)}\right|^{2}+\left|p_{l, m, 2}^{(j)}\right|^{2}+\left|\alpha_{1}^{l} p_{l, m, 2}^{(j)}-\alpha_{2}^{m} p_{l, m, 1}^{(j)}\right|^{2}\right) .
\end{aligned}
$$

It remains to estimate (cf. A.6 and (A.7))

$$
\frac{\left(\alpha_{1}^{l}\right)^{2}+\left(\alpha_{2}^{m}\right)^{2}+\left|\omega^{2} \varepsilon^{(j)} \mu^{(j)}\right|^{2}}{\left|\beta_{l, m}^{(j)}\right|^{2}} \text { and }\left(1+\left(\alpha_{1}^{l}\right)^{2}+\left(\alpha_{2}^{m}\right)^{2}\right)^{1 / 2} \min \left\{2 \delta^{(j)}, \frac{1}{\operatorname{Im}\left(\beta_{l, m}^{(j)}\right)}\right\} \text {. }
$$

First, it follows from (2.13) and the definitions of $\beta_{+}^{(j)}$ and $\beta_{-}^{(j)}$ in (2.45) that

$$
\begin{aligned}
\left|\beta_{l, m}^{(j)}\right|^{2} & =\left(\left(\omega^{2} \operatorname{Im}\left(\varepsilon^{(j)} \mu^{(j)}\right)\right)^{2}+\left(\operatorname{Re}\left(\beta_{l, m}^{(j)}\right)^{2}\right)^{2}\right)^{1 / 2} \\
& \geq\left(\left(\omega^{2} \operatorname{Im}\left(\varepsilon^{(j)} \mu^{(j)}\right)\right)^{2}+\min \left\{\left(\beta_{+}^{(j)}\right)^{4},\left(\beta_{-}^{(j)}\right)^{4}\right\}\right)^{1 / 2}
\end{aligned}
$$


Thus from (2.13),

$$
\begin{aligned}
& \frac{\left(\alpha_{1}^{l}\right)^{2}+\left(\alpha_{2}^{m}\right)^{2}+\left|\omega^{2} \varepsilon^{(j)} \mu^{(j)}\right|^{2}}{\left|\beta_{l, m}^{(j)}\right|^{2}}=\frac{\omega^{2} \operatorname{Re}\left(\varepsilon^{(j)} \mu^{(j)}\right)-\operatorname{Re}\left(\beta_{l, m}^{(j)}\right)^{2}+\left|\omega^{2} \varepsilon^{(j)} \mu^{(j)}\right|^{2}}{\left|\beta_{l, m}^{(j)}\right|^{2}} \\
& \quad \leq \frac{\omega^{2} \operatorname{Re}\left(\varepsilon^{(j)} \mu^{(j)}\right)+\left|\omega^{2} \varepsilon^{(j)} \mu^{(j)}\right|^{2}}{\left|\beta_{l, m}^{(j)}\right|^{2}}+1 \\
& \leq \frac{\omega^{2} \operatorname{Re}\left(\varepsilon^{(j)} \mu^{(j)}\right)+\left|\omega^{2} \varepsilon^{(j)} \mu^{(j)}\right|^{2}}{\left(\left(\omega^{2} \operatorname{Im}\left(\varepsilon^{(j)} \mu^{(j)}\right)\right)^{2}+\min \left\{\left(\beta_{+}^{(j)}\right)^{4},\left(\beta_{-}^{(j)}\right)^{4}\right\}\right)^{1 / 2}}+1 .
\end{aligned}
$$

Secondly, it is clear from (2.46) that $\left|\operatorname{Im}\left(\beta_{l, m}^{(j)}\right)\right|^{2} \geq-\operatorname{Re}\left(\beta_{l, m}^{(j)}\right)^{2}$. Therefore, if $\left(\alpha_{1}^{l}\right)^{2}+\left(\alpha_{2}^{m}\right)^{2}>\omega^{2} \operatorname{Re}\left(\varepsilon^{(j)} \mu^{(j)}\right)+1 /\left(2 \delta^{(j)}\right)^{2}$, then from (2.13),

$$
\begin{aligned}
\frac{1+\left(\alpha_{1}^{l}\right)^{2}+\left(\alpha_{2}^{m}\right)^{2}}{\left|\operatorname{Im}\left(\beta_{l, m}^{(j)}\right)\right|^{2}} & \leq \frac{1+\left(\alpha_{1}^{l}\right)^{2}+\left(\alpha_{2}^{m}\right)^{2}}{\left(\alpha_{1}^{l}\right)^{2}+\left(\alpha_{2}^{m}\right)^{2}-\omega^{2} \operatorname{Re}\left(\varepsilon^{(j)} \mu^{(j)}\right)} \\
& \leq\left(2 \delta^{(j)}\right)^{2}\left(1+\omega^{2} \operatorname{Re}\left(\varepsilon^{(j)} \mu^{(j)}\right)\right)+1 .
\end{aligned}
$$

On the other hand, if $\left(\alpha_{1}^{l}\right)^{2}+\left(\alpha_{2}^{m}\right)^{2} \leq \omega^{2} \operatorname{Re}\left(\varepsilon^{(j)} \mu^{(j)}\right)+1 /\left(2 \delta^{(j)}\right)^{2}$, then

$$
\left(1+\left(\alpha_{1}^{l}\right)^{2}+\left(\alpha_{2}^{m}\right)^{2}\right)\left(2 \delta^{(j)}\right)^{2} \leq\left(2 \delta^{(j)}\right)^{2}\left(1+\omega^{2} \operatorname{Re}\left(\varepsilon^{(j)} \mu^{(j)}\right)\right)+1 .
$$

By combining the above two estimates we deduce

$$
\left(1+\left(\alpha_{1}^{l}\right)^{2}+\left(\alpha_{2}^{m}\right)^{2}\right)^{1 / 2} \min \left\{2 \delta^{(j)}, \frac{1}{\operatorname{Im}\left(\beta_{l, m}^{(j)}\right)}\right\} \leq \sqrt{\left(2 \delta^{(j)}\right)^{2}\left(1+\omega^{2} \operatorname{Re}\left(\varepsilon^{(j)} \mu^{(j)}\right)\right)+1} .
$$

Now, (A.7) follows from plugging (A.13) and (A.14) into (A.12). This completes the proof upon using Lemma 2.2

A.2. Proof of Lemma 4.4. For each face $F \in \mathcal{F}_{h}$ with edges $\left\{e_{1}, e_{2}, e_{3}\right\}$, let $w_{j}, j=1,2,3$ be the nodal basis function of $V_{h}^{1}$ corresponding to $e_{j}$. Suppose that $F$ is the face $f_{123}$ of a tetrahedron element $T$ with vertices $A_{j}, j=1,2,3,4$ and that $e_{1}, e_{2}, e_{3}$ are the edges $A_{2} A_{3}, A_{3} A_{1}, A_{1} A_{2}$, respectively. By using the barycentric coordinates $\left(\lambda_{1}, \lambda_{2}, \lambda_{3}, \lambda_{4}\right), w_{j}, j=1,2,3$ may be expressed as

$$
w_{1}=\lambda_{2} \nabla \lambda_{3}-\lambda_{3} \nabla \lambda_{2}, \quad w_{2}=\lambda_{3} \nabla \lambda_{1}-\lambda_{1} \nabla \lambda_{3}, \quad w_{3}=\lambda_{1} \nabla \lambda_{2}-\lambda_{2} \nabla \lambda_{1} .
$$

We construct a dual basis $\left\{\phi_{k}\right\}$ of $\left\{w_{i} \times \nu\right\}$ as follows:

$$
\int_{F}\left(w_{j} \times \nu\right) \cdot \phi_{k}=\delta_{j k}, \quad j, k=1,2,3 .
$$

Here $\nu$ is a unit normal vector to $F$. We claim that

$$
\left\|\phi_{k}\right\|_{\left(L^{\infty}(F)\right)^{3}} \leq C h_{F}^{-1} \quad \text { and hence } \quad\left\|\phi_{k}\right\|_{\left(L^{2}(F)\right)^{3}} \leq C .
$$

Without loss of generality, we shall prove that (A.16) holds for $k=1$. We find $y=\left(y_{1}, y_{2}, y_{3}\right)^{\mathrm{T}}$ such that

$$
\phi_{1}=y_{1} w_{1} \times \nu+y_{2} w_{2} \times \nu+y_{3} w_{3} \times \nu, \quad \int_{F}\left(w_{j} \times \nu\right) \cdot \phi_{1}=\delta_{j 1}, \quad j=1,2,3 .
$$


It is clear that $y$ is the solution of the linear system:

$$
A_{F} y=(1,0,0)^{\mathrm{T}}, \quad \text { where } A_{F}=\left(\int_{F}\left(w_{j} \times \nu\right) \cdot\left(w_{k} \times \nu\right)\right)_{3 \times 3} .
$$

We shall show that $A_{F}$ is invertible.

Let $b_{j k}=\left(\nabla \lambda_{j} \times \nu\right) \cdot\left(\nabla \lambda_{k} \times \nu\right)$. Since $\sum_{j=1}^{4} \nabla \lambda_{j}=0$ and $\nabla \lambda_{4}$ is perpendicular to the face $f_{123}$, we have, for $j, k=1,2,3$,

$$
\sum_{k=1}^{3} b_{j k}=0 \quad \text { and } \quad b_{j k}=b_{k j}
$$

Therefore, $A_{F}$ can be rewritten as

$$
A_{F}=\frac{|F|}{12}\left(\begin{array}{ccc}
3 b_{22}+3 b_{33}-b_{11} & -3 b_{33}+b_{11}+b_{22} & -3 b_{22}+b_{33}+b_{11} \\
-3 b_{33}+b_{11}+b_{22} & 3 b_{11}+3 b_{33}-b_{22} & -3 b_{11}+b_{22}+b_{33} \\
-3 b_{22}+b_{33}+b_{11} & -3 b_{11}+b_{22}+b_{33} & 3 b_{11}+3 b_{22}-b_{33}
\end{array}\right) .
$$

It follows from $\nabla \lambda_{1} \perp f_{234}$ that

$$
\left|\nabla \lambda_{1}\right|=1 / \text { the height of } T \text { to the face } f_{234},
$$

which implies that

$$
b_{11}=\left|\nabla \lambda_{1} \times \nu\right|^{2}=\frac{\left|e_{1}\right|^{2}}{4|F|^{2}} . \quad \text { Similarly, } \quad b_{22}=\frac{\left|e_{2}\right|^{2}}{4|F|^{2}}, \quad b_{33}=\frac{\left|e_{3}\right|^{2}}{4|F|^{2}} .
$$

Straightforward computations show that

$$
\operatorname{det} A_{F}=\frac{\left|e_{1}\right|^{2}+\left|e_{2}\right|^{2}+\left|e_{3}\right|^{2}}{576|F|} \geq c_{0},
$$

where $c_{0}$ is a positive constant depending only on the minimum angle of the elements in the mesh. Thus $A_{F}$ is invertible. Since $A_{F}=O(1)$, we have $A_{F}^{-1}=O(1)$, which implies that $y=A_{F}^{-1}(1,0,0)^{\mathrm{T}}=O(1)$, i.e., (A.16) holds.

Now for each edge $e$, we assign one of those faces with edge $e$ and call it $F_{e}$. We have to comply with the restriction that for $e$ on the boundary, $F_{e}$ also on the boundary, and that if $e$ is on $\Gamma_{10}$ (or $\Gamma_{20}$ ) and $e^{\prime}$ is the corresponding edge on $\Gamma_{11}$ (or $\Gamma_{21}$ ), then $F_{e^{\prime}}$ is also the corresponding face of $F_{e}$. Then we can define

$$
Q_{h} \psi^{(1)}=\sum_{e \in \mathcal{E}_{h}}\left(\int_{F_{e}}\left(\psi^{(1)} \times \nu\right) \cdot \phi_{e}^{F_{e}}\right) w_{e},
$$

where $\mathcal{E}_{h}$ is the set of all edges in $\mathcal{M}_{h}, w_{e}$ is the basis function corresponding to the edge $e$, and $\phi_{e}^{F_{e}}$ is the dual basis function corresponding to $w_{e}$. By virtue of (A.15) this defines a projection. Obviously the boundary condition is respected. Let $T_{e} \in \mathcal{M}_{h}$ be an element with $F_{e}$ as one of its faces. Then

$$
\left|\int_{F_{e}}\left(\psi^{(1)} \times \nu\right) \cdot \phi_{e}^{F_{e}}\right| \leq\left\|\psi^{(1)}\right\|_{\left(L^{2}\left(F_{e}\right)\right)^{3}}\left\|\phi_{e}^{F_{e}}\right\|_{\left(L^{2}\left(F_{e}\right)\right)^{3}} \leq C\left\|\psi^{(1)}\right\|_{\left(L^{2}\left(F_{e}\right)\right)^{3}},
$$

where we have used (A.16). By the scaled trace inequality, we have

$$
\left\|\psi^{(1)}\right\|_{\left(L^{2}\left(F_{e}\right)\right)^{3}}^{2} \leq C\left(h_{e}^{-1}\left\|\psi^{(1)}\right\|_{\left(L^{2}\left(T_{e}\right)\right)^{3}}^{2}+h_{e}\left|\psi^{(1)}\right|_{\left(H^{1}\left(T_{e}\right)\right)^{3}}^{2}\right) .
$$


Therefore,

$$
\begin{aligned}
\left\|Q_{h} \psi^{(1)}\right\|_{\left(L^{2}(T)\right)^{3}}^{2} & \leq C h_{T} \sum_{\substack{e \in \mathcal{E}_{h} \\
e \subset \partial T}}\left|\int_{F_{e}}\left(\psi^{(1)} \times \nu\right) \phi_{e}^{F_{e}}\right|^{2} \\
& \leq C h_{T} \sum_{\substack{e \in \mathcal{E}_{h} \\
e \subset \partial T}}\left(h_{e}^{-1}\left\|\psi^{(1)}\right\|_{\left(L^{2}\left(T_{e}\right)\right)^{3}}^{2}+h_{e}\left|\psi^{(1)}\right|_{\left(H^{1}\left(T_{e}\right)\right)^{3}}^{2}\right) \\
& \leq C\left(\left\|\psi^{(1)}\right\|_{\left(L^{2}(\widetilde{T})\right)^{3}}^{2}+h_{T}^{2}\left|\psi^{(1)}\right|_{\left(H^{1}(\widetilde{T})\right)^{3}}^{2}\right) .
\end{aligned}
$$

This proves the first estimate in the lemma.

Since $Q_{h}$ is a projection, we know that $Q_{h} c_{T}=c_{T}$ for any constant $c_{T}$. Thus

$$
\begin{aligned}
& \left\|\psi^{(1)}-Q_{h} \psi^{(1)}\right\|_{L^{2}(T)}=\inf _{c_{T}}\left\|\left(\psi^{(1)}+c_{T}\right)-Q_{h}\left(\psi^{(1)}+c_{T}\right)\right\|_{L^{2}(T)} \\
& \leq C \inf _{c_{T}}\left(\left\|\psi^{(1)}+c_{T}\right\|_{\left(L^{2}(\widetilde{T})\right)^{3}}+h_{T}\left|\psi^{(1)}+c_{T}\right|_{\left(H^{1}(\widetilde{T})\right)^{3}}\right) \leq C h_{T}\left|\psi^{(1)}\right|_{\left(H^{1}(\widetilde{T})\right)^{3}},
\end{aligned}
$$

where we have used the Bramble-Hilbert Lemma to derive the last inequality. This proves (4.8). The estimate (4.9) is a direct consequence of (4.8) by the scaled trace inequality.

\section{REFERENCES}

1. T. Abboud, Formulation variationnelle des équations de Maxwell dans un réseau bipériodique de $\mathbf{R}^{3}$, C. R. Acad. Sci. Paris, Série I 317 (1993), 245-248. MR1233420 (94f:78002)

2. H. Ammari and G. Bao, Maxwell's equations in periodic chiral structures, Math. Nachr. 251 (2003), 3-18. MR.1960800 (2004a:78013)

3. H. Ammari and J. Nédélec, Low-frequency electromagnetic scattering, SIAM J. Math. Anal. 31 (2000), 836-861. MR1752419 (2001b:78006)

4. D.N. Arnold, A. Mukherjee, and L. Pouly, Locally adapted tetrahedral meshes using bisection, SIAM J. Sci. Comput. 22 (2000), 431-448. MR1780608(2002h:65204)

5. I. Babuška and A. Aziz, Survey lectures on the mathematical foundations of the finite element method. in The mathematical foundations of the finite element method with applications to partial differential equations, Academic Press, New York, 1972. MR0421106 (54:9111)

6. I. Babuška and W.C. Rheinboldt, Error estimates for adaptive finite element computations, SIAM J. Numer. Anal. 15 (1978), 736-754. MR0483395 (58:3400)

7. G. Bao, Variational approximation of Maxwell's equations in biperiodic structures, SIAM J. Appl. Math. 57 (1997), 364-381. MR1438758 (97m:65199)

8. G. Bao, Z. Chen, and $\mathrm{H}$. Wu, Adaptive finite-element method for diffraction gratings, J. Opt. Soc. Amer. A 22 (2005), 1106-1114. MR2188104

9. G. Bao, L. Cowsar, and W. Masters, Mathematical modeling in optical science, Frontiers Appl. Math., vol. 22, SIAM, Philadelphia, 2001. MR.1831328 (2001m:78002)

10. G. Bao and D.C. Dobson, On the scattering by a biperiodic structure, Proc. Amer. Math. Soc. 128 (2000), 2715-2723. MR.1694448 (2000m:35051)

11. G. Bao, D.C. Dobson, and J.A. Cox, Mathematical studies in rigorous grating theory, J. Opt. Soc. Amer. A 12 (1995), 1029-1042. MR1335399 (96b:78028)

12. G. Bao and $\mathrm{H}$. Wu, Convergence analysis of the PML problems for time-harmonic Maxwell's equations, SIAM J. Numer. Anal. 43 (2005), 2121-2143. MR2192334 (2007h:65115)

13. G. Bao and A. Zhou, Analysis of finite dimensional approximations to a class of partial differential equations, Math. Meth. Appl. Sci. 27 (2004), 2055-2066. MR2099817(2005i:65085)

14. R. Beck, R. Hiptmair, R. Hoppe, and B. Wohlmuth, Residual based a posteriori error estimators for eddy current computation, M2AN Math. Model Numer. Anal. 34 (2000), 159-182. MR:1735971 (2000k:65203)

15. J.-P. Berenger, A perfectly matched layer for the absorption of electromagnetic waves, J. Comput. Phys. 114 (1994), 185-200. MR.1294924 (95e:78002) 
16. - Three-dimensional perfectly matched layer for the absorption of electromagnetic waves, J. Comput. Phys. 127 (1996), 363-379. MR1412240 (97h:78001)

17. P. Binev, W. Dahmen and R. DeVore, Adaptive finite elements with convergence rates, Numer. Math. 97 (2004), 219-268. MR.2050077 (2005d:65222)

18. J. Bramble and J. Pasciak, Analysis of a finite PML approximation for the three dimensional time-harmonic Maxwell and acoustic scattering problems, Math. Comp. 76 (2007), 597-614. MR.2291829 (2008b:65130)

19. - Analysis of a finite element PML approximation for the three dimensional timeharmonic Maxwell problem., Math. Comp. 77 (2008), 1-10. MR.2353940 (2008i:65250)

20. Y. Chang, G. Li, H. Chu, and J. Opsal, Efficient finite-element, Green's function approach for critical-dimension metrology of three-dimensional gratings on multilayer films, J. Opt. Soc. Amer. A 23 (2006), 638-645. MR 2232900

21. Z. Chen and J. Chen, An adaptive perfectly matched layer technique for 3-D timeharmonic electromagnetic scattering problems, Math. Comp. 77 (2008), 673-698. MR2373175 (2008m:78007)

22. Z. Chen and S. Dai, On the efficiency of adaptive finite element methods for elliptic problems with discontinuous coefficients, SIAM J. Sci. Comput. 24 (2002), 443-462. MR1951050 (2003k:65160)

23. Z. Chen and X. Liu, An adaptive perfectly matched layer technique for time-harmonic scattering problems., SIAM J. Numer. Anal. 43 (2005), 645-671. MR.2177884 (2006m:65260)

24. Z. Chen and $\mathrm{H}$. Wu, An adaptive finite element method with perfectly matched absorbing layers for the wave scattering by periodic structures, SIAM J. Numer. Anal. 41 (2003), 799826. MR2005183 (2004k:65215)

25. F. Collino and P. Monk, Optimizing the perfectly matched layer, Comput. Methods Appl. Mech. Engrg. 164 (1998), 157-171. MR1662777(99h:78002)

26. M. Costabel and M. Dauge, Maxwell and Lamé eigenvalues on polyhedra, Math. Meth. Appl. Sci. 164 (1999), 243-258. MR 1672271 (99k:78009)

27. D. Dobson and A. Friedman, The time-harmonic Maxwell equations in a doubly periodic structure, J. Math. Anal. Appl. 166 (1992), 507-528. MR1160941 (92m:78015)

28. W. Dörfler, A convergent adaptive algorithm for Poisson's equation, SIAM J. Numer. Anal. 33 (1996), 1106-1124. MR 1393904 (97e:65139)

29. R.E. Edwards, Fourier series, a modern introduction, vols. 1, 2, Springer-Verlag, New York, 1979, 1982. MR545506 (80j:42001); MR0667519 (83k:42001)

30. S.D. Gedney, An anisotropic perfectly matched layer absorbing medium for the truncation of FDTD lattices, IEEE Trans. Antennas Propagat. 44 (1996), 1630-1639.

31. V. Girault and P.A. Raviart, Finite element methods for Navier-Stokes equations: Theory and algorithms, Springer-Verlag, New York, 1986. MR851383 (88b:65129)

32. M. Lassas and E. Somersalo, On the existence and convergence of the solution of PML equations, Computing 60 (1998), 229-241. MR1621305 (99a:65133)

33. L. Li, New formulation of the Fourier modal method for crossed surface-relief gratings, J. Opt. Soc. Amer. A 14 (1997), 2758-2767.

34. K. Mekchay and R. Nochetto, Convergence of adaptive finite element methods for general second order linear elliptic PDES, SIAM J. Numer. Anal. 43 (2005), 1803-1827. MR2192319 (2006i:65201)

35. P. Monk, A posteriori error indicators for Maxwell's equations, J. Comput. Appl. Math. 100 (1998), 173-190. MR1659117 (2000k:78020)

36. , Finite element methods for Maxwell's equations, Clarendon Press, Oxford, 2003. MR2059447 (2005d:65003)

37. P. Monk and E. Süli, The adaptive computation of far-field patterns by a posteriori error estimation of linear functionals, SIAM J. Numer. Anal. 36 (1999), 251-274. MR 1654563 (2000a:65137)

38. P. Morin, R.H. Nochetto, and K.G. Siebert, Data oscillation and convergence of adaptive finite element methods, SIAM J. Numer. Anal. 38 (2000), 466-488. MR.1770058 (2001g:65157)

39. — Convergence of adaptive finite element methods, SIAM Review 44 (2002), no. 4, 631-658. MR.1980447

40. J.-C. Nédélec, Mixed finite elements in $R^{3}$, Numer. Math. 35 (1980), 315-341. MR592160 (81k:65125) 
41. R. Petit, Electromagnetic theory of gratings, Topics in Current Physics, vol. 22, SpringerVerlag, Heidelberg, 1980. MR609533 (82a:78001)

42. H.J. Schmeisser and H. Triebel, Topics in Fourier analysis and function spaces, John Wiley \& Sons, New York, 1987. MR.891189 (88k:42015b)

43. L.R. Scott and S. Zhang, Finite element interpolation of nonsmooth functions satisfying boundary conditions, Math. Comp. 54 (1990), 483-493. MR1011446 (90j:65021)

44. R. Stevenson, Optimality of a standard adaptive finite element method., Found. Comput. Math. 7 (2007), 245-269. MR2324418 (2008i:65272)

45. F. Teixera and W. Chew, Systematic derivation of anisotropic PML absorbing media in cylindrical and spherical coordinates, IEEE microwave and guided wave letters 7 (1997), 371373.

46. E. Turkel and A. Yefet, Absorbing PML boundary layers for wave-like equations, Appl. Numer. Math. 27 (1998), 533-557. MR1644675 (99g:78008)

47. R. Verfürth, A review of a posteriori error estimation and adaptive mesh refinement techniques, Teubner, Stuttgart, 1996.

48. H. Wu and Z. Zhang, Can we have superconvergent gradient recovery under adaptive meshes?, SIAM J. Numer. Anal. 45 (2007), 1701-1722. MR2338406 (2008k:65252)

49. V. Yachin and K. Yasumoto, Method of integral functionals for electromagnetic wave scattering from a double-periodic magnetodielectric layer, J. Opt. Soc. Amer. A 24 (2007), 36063618 .

Department of Mathematics, Michigan State University, East Lansing, Michigan 48824

E-mail address: bao@math.msu.edu

Department of Mathematics, Purdue University, West Lafayette, Indiana 47907

E-mail address: lipeijun@math.purdue.edu

Department of Mathematics, Nanjing University, Jiangsu, 210093, China

E-mail address: hjw@nju.edu.cn 